\title{
Adaptive PSF fitting - a highly performing photometric method and light curves of the GLS H1413+117: time delays and micro-lensing effects
}

\author{
T. A. Akhunov, ${ }^{1,2,3 \star}$ O. Wertz, ${ }^{3 \star}$ A. Elyiv, ${ }^{3,4,5 \star ~ R . ~ G a i s i n, ~}{ }^{1}$ B. P. Artamonov, ${ }^{6}$ \\ V. N. Dudinov, ${ }^{7,8}$ S. N. Nuritdinov, ${ }^{2}$ C. Delvaux, ${ }^{3,9}$ A. V. Sergeyev, ${ }^{7,8}$ A. S. Gusev, ${ }^{6}$ \\ V. V. Bruevich, ${ }^{6}$ O. Burkhonov, ${ }^{1}$ A. P. Zheleznyak, ${ }^{7,8}$ O. Ezhkova ${ }^{6}$ and J. Surdej ${ }^{3}$ \\ ${ }^{1}$ Ulugh Beg Astronomical Institute of the Uzbek Academy of Sciences, Astronomicheskaya 33, 100052 Tashkent, Uzbekistan \\ ${ }^{2}$ National University of Uzbekistan, Physics Faculty, Department of Astronomy and Atmospheric Physics, 100174 Tashkent, Uzbekistan \\ ${ }^{3}$ Institut d'Astrophysique et de Géophysique, Université de Liège, Allée du 6 Août, 19c, Sart Tilman, B-4000 Liège, Belgium \\ ${ }^{4}$ Main Astronomical Observatory, Academy of Sciences of Ukraine, 27 Akademika Zabolotnoho St., UA-03680 Kyiv, Ukraine \\ ${ }^{5}$ Dipartimento di Fisica e Astronomia, Universitá di Bologna, viale Berti Pichat 6/2, I-40127 Bologna, Italy \\ ${ }^{6}$ Sternberg Astronomical Institute (SAI), Lomonosov Moscow State University, Universitetskii pr. 13, 119992 Moscow, Russia \\ ${ }^{7}$ Institute of Radio Astronomy, Krasnoznamennaya 4, UA-61002 Kharkov, Ukraine \\ ${ }^{8}$ Institute of Astronomy of Kharkov National University, Sumskaya 35, UA-61022 Kharkov, Ukraine \\ ${ }^{9}$ Max-Planck-Institut für extraterrestrische Physik, Giessenbachstrasse 1, D-85748 Garching, Germany
}

Accepted 2016 November 14. Received 2016 November 1; in original form 2015 March 22

\begin{abstract}
We present new photometric observations of $\mathrm{H} 1413+117$ acquired during seasons between 2001 and 2008 in order to estimate the time delays between the lensed quasar images and to characterize at best the on-going micro-lensing events. We propose a highly performing photometric method called the adaptive point spread function fitting and have successfully tested this method on a large number of simulated frames. This has enabled us to estimate the photometric error bars affecting our observational results. We analysed the $V$ - and $R$-band light curves and $V-R$ colour variations of the A-D components which show short- and long-term brightness variations correlated with colour variations. Using the $\chi^{2}$ and dispersion methods, we estimated the time delays on the basis of the $R$-band light curves over the seasons between 2003 and 2006. We have derived the new values: $\Delta t_{\mathrm{AB}}=-17.4 \pm 2.1, \Delta t_{\mathrm{AC}}=-18.9 \pm 2.8$ and $\Delta t_{\mathrm{AD}}=28.8 \pm 0.7 \mathrm{~d}$ using the $\chi^{2}$ method ( $\mathrm{B}$ and $\mathrm{C}$ are leading, $\mathrm{D}$ is trailing) with $1 \sigma$ confidence intervals. We also used available observational constraints (resp. the lensed image positions, the flux ratios in mid-IR and two sets of time delays derived in the present work) to update the lens redshift estimation. We obtained $z_{1}=1.95_{-0.10}^{+0.06}$ which is in good agreement with previous estimations. We propose to characterize two kinds of micro-lensing events: micro-lensing for the A, B, C components corresponds to typical variations of $\sim 10^{-4} \mathrm{mag} \mathrm{d}^{-1}$ during all the seasons, while the $\mathrm{D}$ component shows an unusually strong micro-lensing effect with variations of up to $\sim 10^{-3} \mathrm{mag} \mathrm{d}^{-1}$ during 2004 and 2005 .
\end{abstract}

Key words: gravitational lensing: strong-gravitational lensing: micro-methods: data analysis - techniques: photometric: individual: H1413+117.

\section{INTRODUCTION}

Gravitational lensing offers a unique tool to study the mass distribution of the lens, the structure of the light source as well as the geometry of the Universe. However, any significant contribution to these endeavours requires some continuous and detailed investigations of each known gravitational lens system. One example of such a long-term investigation of a gravitationally lensed

\footnotetext{
^E-mail: talat77@ rambler.ru (TAA); jsurdej@ulg.ac.be (OW); owertz@
} alumni.ulg.ac.be (AE) quasar is $\mathrm{H} 1413+117$, also known as the Clover Leaf, whose spectrum was first observed by Hazard et al. (1984) and Drew \& Boksenberg (1984). Later it became evident that this object is a lensed quasar displaying four images (Magain et al. 1988). This source is relatively distant $(z \approx 2.55)$ and its brightness classifies it as a highly luminous quasar $\left(M_{V}<-29\right.$ and $\left.m_{V} \approx 17\right)$, while its spectrum revealed it to be a broad absorption line quasar (Hazard et al. 1984). The four lensed images display an almost symmetric configuration, the maximum angular separation between them being 1.36 arcsec. But the nature of the lensing body still remains open.

On the basis of the comparison between optical and radio observations, two potential gravitational lens models of the Clover 
Leaf have been proposed: (a) a single elliptical galaxy or (b) two spherical galaxies. Theoretical estimations of the time delays have led to values of about several weeks (Kayser et al. 1990). The first light curves of the individual lensed images of $\mathrm{H} 1413+117$ in the Bessel $V$ filter have been reported by Arnould et al. (1993). They very sparsely cover the time interval between 1987 and 1993. The light curves of the individual components varied almost simultaneously, which indicates that the time delays should be much shorter than the sampling period ( $\sim 1 \mathrm{yr}$ ). Subsequent re-analysis (Remy et al. 1996) of the same data led to some more elaborated conclusions. Namely, (a) the fluxes of the A, B, C components vary in parallel and almost simultaneously; (b) the expected time delays between the lensed components must be shorter than 1 month. On the other side, the weakest D component showed additional light variations besides the global trend, which was the first evidence reported for a micro-lensing event in the H1413+117 system (Remy et al. 1996).

In a series of papers (Turnshek et al. 1988, 1997; Monier, Turnshek \& Lupie 1998), detailed studies of the optical images and spectra of the lensed quasar H1413+117 obtained with the HST and Multiple Mirror Telescope were carried out. A faint elongated arc between the $\mathrm{A}$ and $\mathrm{C}$ components seems to indicate the presence of an Einstein ring-like structure (Turnshek et al. 1997). According to Turnshek et al. (1997), any future model of this system must take into account the different colours of the components, i.e. the line-ofsight-dependent extinction. In addition to three metal-line systems of absorption lines at $z_{\mathrm{abs}}=1.66,1.44$ and 2.07 (Hazard et al. 1984; Magain et al. 1988), numerous absorption lines were found at $z_{\text {abs }}$ $=0.61 \div 2.1$ (Turnshek et al. 1988; Monier et al. 1998). The lensing galaxy had not been detected, but its redshift was expected to be $z_{1}$ $>1$. Otherwise, the lens would have been detected on the HST images (Turnshek et al. 1988; Monier et al. 1998). Using astrometric and photometric data obtained by Turnshek et al. (1997), Chae \& Turnshek (1999) constrained at best plausible lens models for the Clover Leaf. Another group showed that an external shear is needed to correctly model this system (Keeton, Kochanek \& Seljak 1997).

Chae et al. (2001) found new pieces of evidence for micro-lensing of the D component and showed that this component significantly displays different relative polarization properties compared to the three other ones. More recently, additional numerous spectroscopic pieces of evidence of micro-lensing were reported for the components of the Clover Leaf. Most micro-lensing activity is seen for the D component, which variability lasts for at least several years (Chartas et al. 2004; Jovanović 2005). Moreover, the micro-lensing of the D component shows a chromatic dependence (Hutsemékers et al. 2010). This result is directly consistent with the estimation of the duration of the micro-lensing events in $\mathrm{H} 1413+117$ by Witt, Mao \& Schechter (1995).

The lensing body of $\mathrm{H} 1413+117$ represents another difficulty. During a long time, this has only been investigated theoretically. The first observational conclusions about this deflector were provided by Kneib et al. (1998a) and Kneib, Alloin \& Pello (1998b). They estimated the brightness of the lensing galaxy to be $m_{160 \mathrm{w}} \approx$ 20.6, and concluded that it may belong to a galaxy cluster with a redshift $z_{\text {cluster }} \approx 1.7$. These results were later supported by Chantry \& Magain (2007), who confirmed that the lensing galaxy is a really diffuse and faint object: $m_{160 \mathrm{w}}=20.5$ and $m_{180 \mathrm{w}}=22.2$. Those conclusions were enriched by the precise astrometry of the lensed components of the system via mid-infrared and modelling investigations by MacLeod, Kochanek \& Agol (2009).

Finally, we should note that the only work dedicated to the observational determination of the time delays between the components

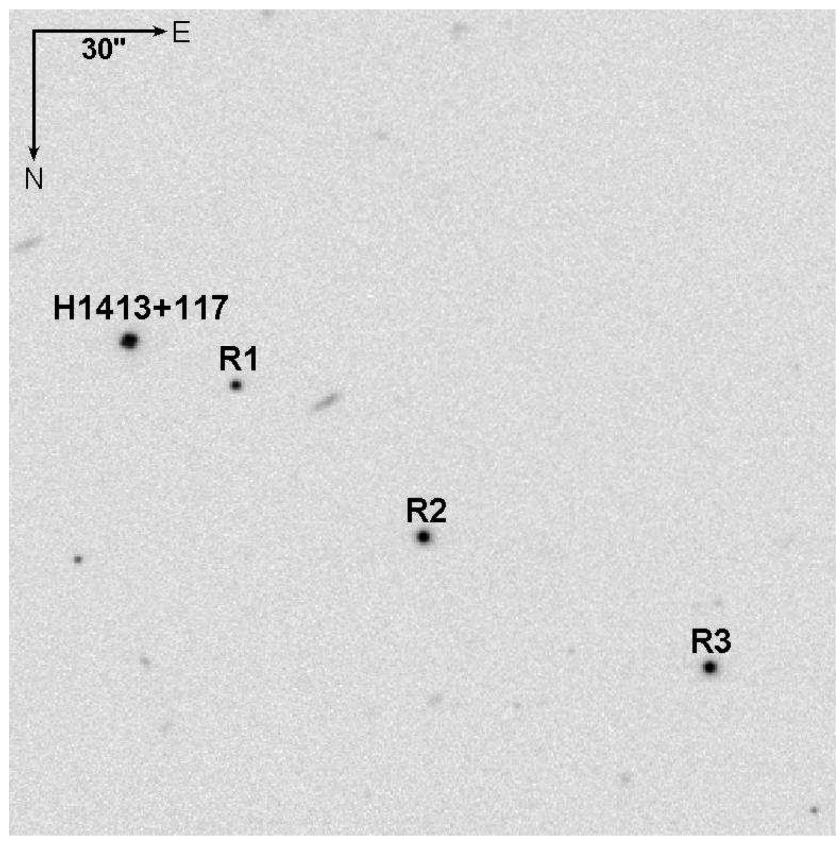

Figure 1. $R$-band CCD frame of the GLS H1413+117 obtained with the Maidanak 1.5-m telescope, on 2003 February 10. The quasar and the reference stars R1, R2 and R3 are labelled. The field angular size is 2.2 $\operatorname{arcmin} \times 2.2$ arcmin. The stars R1 and R2 are named as Objects 40 and 45 in table 2 of the paper by Kayser et al. (1990), respectively.

of the Clover Leaf has been performed by Goicoechea \& Shalyapin (2010). Using $r$ Sloan band observations in 2008, they were able to predict the values of the time delays between three pairs of components: $\Delta t_{\mathrm{AB}}=-17 \pm 3, \Delta t_{\mathrm{AC}}=-20 \pm 4, \Delta t_{\mathrm{AD}}=23 \pm 4 \mathrm{~d}$ (where $\mathrm{A}, \mathrm{B}$ and $\mathrm{C}$ are leading, and $\mathrm{D}$ is the trailing component). Moreover, those authors, using their new time delay constraints, improved the lens model and estimated the possible redshift of the lensing galaxy: $z_{1}=1.88_{-0.11}^{+0.09}$.

In this paper, we present the results of long-term monitoring observations of $\mathrm{H} 1413+117$ carried out at the Maidanak observatory (Uzbekistan) between 2001 and 2008 (in the $R$ and $V$ bands). The details of the monitoring program and the observational data are presented in Section 2. The photometric technique applied here the adaptive point spread function (PSF) fitting method - is described in Section 3. Further, in Sections 4 and 5, we detail the application of this new method to the lensed images of the quasar. Using these new observations and the analysis of the variations of the four component light curves, we determine the time delay values between the six possible pairs of lensed images (Section 6). The light and colour curves and the micro-lensing analysis are described in Section 7. In Section 8, we adopt the lensing scenario proposed in MacLeod et al. (2009) and Goicoechea \& Shalyapin (2010) to update the estimate of the lens redshift. To this aim, we adopt a more recent value for the Hubble parameter and a set of time delays derived in the present paper. A summary and conclusions are given in Section 9.

\section{OBSERVATIONS}

The observational data of $\mathrm{H} 1413+117$ (see Figs 1 and 2) were collected between 2001 and 2008 during a quasar monitoring programme carried out by the Maidanak Gravitational Lens System (GLS) collaboration. CCD frames in the Bessel $R$ and $V$ bands 


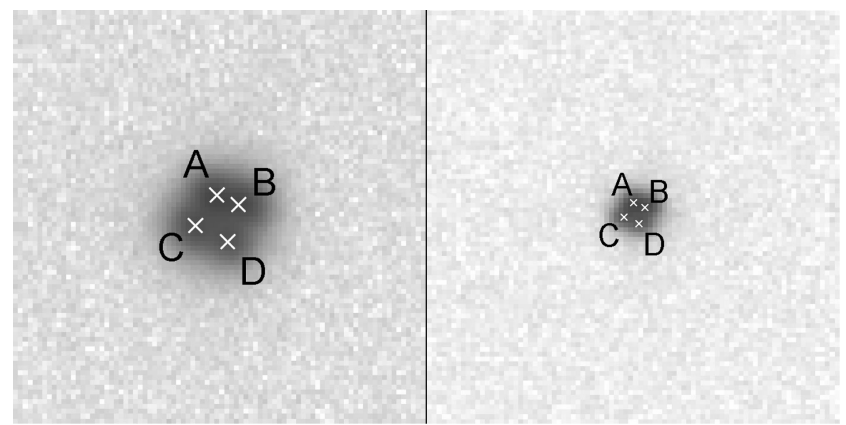

Figure 2. Zoomed $R$-band images of the central parts of H1413+117. Left: long-focus mode (pixel size is $0.135 \mathrm{arcsec} ; 2001$ May 21). The seeing is $\sim 0.73 \mathrm{arcsec}$ and the image size is $13.5 \operatorname{arcsec} \times 13.5$ arcsec. Right: shortfocus mode (pixel size is 0.265 arcsec; 2003 February 10). The seeing is $\sim 0.66$ arcsec and the image size is $26.5 \operatorname{arcsec} \times 26.5 \operatorname{arcsec}$. Orientation: North is down, East to the right.

Table 1. Summary of the H1413+117 Clover Leaf observational data collected during the seasons from 2001 to 2008 (under good observing conditions).

\begin{tabular}{lcccc}
\hline Season & $\begin{array}{c}R \text { band } \\
\text { Period }\end{array}$ & $\mathrm{NN}^{a}$ & Period & $\mathrm{NN}^{a}$ \\
\hline 2001 & $21 / 05$ & 1 & $21 / 05$ & 1 \\
2002 & $3 / 03$ to $9 / 05$ & 6 & $26 / 03$ to $9 / 04$ & 2 \\
2003 & $28 / 01$ to $31 / 07$ & 20 & $7 / 05$ to $19 / 07$ & 10 \\
2004 & $22 / 01$ to $4 / 08$ & 32 & $26 / 02$ to $4 / 08$ & 22 \\
2005 & $8 / 02$ to $30 / 06$ & 20 & $13 / 07$ & 1 \\
2006 & $4 / 01$ to $18 / 07$ & 20 & - & - \\
2007 & $25 / 05$ to $17 / 07$ & 4 & - & - \\
2008 & $9 / 05$ to $4 / 06$ & 5 & - & - \\
\hline
\end{tabular}

Period given in $\mathrm{dd} / \mathrm{mm}$.

${ }^{a} \mathrm{NN}$ - number of nights.

were obtained with the 1.5-m AZT-22 telescope of the Maidanak Observatory, Uzbekistan (Ehgamberdiev et al. 2000; Artamonov et al. 2010), using two different CCD cameras. During all seasons except 2007, the frames were obtained with the $2000 \times 800$ pixel SITe-005 CCD camera. Among them, the CCD frames of 2001 and 2002 were taken in the long-focus mode, while the other ones were taken in the short-focus mode. The angular pixel sizes are 0.135 and 0.267 arcsec, respectively. CCD frames in 2007 were taken with the $4096 \times 4096$ SNUCAM (provided by Seoul University CAMera) camera in the short-focus mode, which has a pixel scale of 0.267 arcsec and a field of view of $18.1 \times 18.1$ arcmin. The photometric filters used were the $R$ and $V$ filters of the JohnsonCousins filter system. We observed on average six frames per night, with an exposure time of $180 \mathrm{~s}$ in $R$ and $210 \mathrm{~s}$ in $V$. A summary of the observational data acquired between 2001 and 2008 is given in Table 1.

Our data cover eight observational seasons and consist of 202 CCD frames in the $R$-filter and 54 CCD frames in the $V$ filter (each of these frames is the result of an average combination of the frames which were obtained during the given night). However, a careful analysis of the data forced us to reject frames obtained under bad observing conditions: a seeing $>1.2$ arcsec and a sky background value $>2000 \mathrm{ADU} \mathrm{pixel}^{-1}$. Nevertheless, in some cases of excellent seeing, we accepted the frames affected by a sky background value as large as $4200 \mathrm{ADU}_{\text {pixel }^{-1}}$. Finally, we are left with 108 frames in the $R$ filter and 35 frames in the $V$ filter. The average seeing and signal-to-noise ratio measured for a field star R1 having a brightness similar to those of the quasar images are 1.03 arcsec and 131.8, respectively. These data are given in Table 2 and Appendix A.

The pre-treatment applied to the Maidanak data consisting of the standard procedure (bias correction and flat-fielding) was done using exclusively the standard IRAF software. The results of the photometry of the lensed images and their analysis are presented in the next sections.

\section{THE ADAPTIVE PSF-FITTING PHOTOMETRIC METHOD}

Several methods have been developed to measure the flux of multiple sources with a small separation (e.g. Stetson, Davis \& Crabtree 1990; Magain, Courbin \& Sohy 1998; Lupton et al. 2001; Koptelova et al. 2005). The majority of these methods requires the assumption of PSF invariance over the frame. This postulate imposes some restrictions on the photometric methods, as the observing conditions or the imaging system do not always encounter this assumption. We propose a new approach to overcome this limitation.

The adaptive PSF-fitting method is an efficient photometric technique that aims at determining the PSF from the lensed components themselves. The fluxes of the images are needed to accurately determine the PSF, but they also need the PSF to be accurately calculated. Consequently, this method constitutes an iterative process in order to calculate both of them.

Let us consider a CCD frame consisting of $M \times N$ pixels. The flux distribution of the source is represented by $D(i, j)$, and the average value of the sky background by $S_{\mathrm{b}}$. The corresponding pixel flux uncertainty is then defined as $\sigma(i, j)$ :

$\sigma(i, j)=\frac{\sqrt{\left[D(i, j)+S_{\mathrm{b}}\right] \cdot \mathrm{gn}^{2}+\mathrm{rd}^{2}}}{\mathrm{gn}}$

where $\mathrm{rd}$ is the read-out noise $\left(\mathrm{e}^{-}\right)$and $\mathrm{gn}\left(\mathrm{e}^{-} \mathrm{ADU}^{-1}\right)$ the gain of the CCD. Here $i \in\{1, \cdot, M\}$ and $j \in\{1, \cdot \cdot N\}$. Let us assume that a number of $K$ point-like sources contribute to $D(i, j)$. In our case, these point-like sources correspond to the four lensed quasar (QSO) images of H1413+117. Each lensed component may be represented by the unknown $\operatorname{PSF}(i, j)$ centred at the position $\left(i_{k}, j_{k}\right)$ with a central intensity $I_{k}$ :

$D(i, j)=\sum_{k}^{K} I_{k} \cdot \operatorname{PSF}\left(i-i_{k}, j-j_{k}\right)+\varepsilon(i, j)$,

where $\varepsilon(i, j)$ represents the noise contribution. The adaptive PSFfitting method consists in reducing the $\chi^{2}$ between $D(i, j)$ and the sum of all the scaled PSFs by adjusting the values of the $I_{k}$ and $\operatorname{PSF}(i, j)$ parameters until reaching convergence. The corresponding $\chi^{2}$ function is defined as:

$\chi^{2}=\sum_{i=1, j=1}^{M, N} \frac{\left[\sum_{k=1}^{K} I_{k} \cdot \operatorname{PSF}\left(i-i_{k}, j-j_{k}\right)-D(i, j)\right]^{2}}{\sigma^{2}(i, j)}$.

To determine both sets of parameters, it is necessary to alternatively calculate them: adopting initial values for the first set of parameters, the second set can be calculated by minimizing the $\chi^{2}$. Then this second set is used to re-calculate the first set of parameters, which will be re-used to determine the values of the second one, etc. Since we are attempting to analyse a frame in order to extract some flux information, it is simpler to provide a relevant initial PSF than providing relevant initial central intensities. Let us assume that we 
Table 2. Photometry of $\mathrm{H} 1413+117$ in the $R$ and $V$ bands. Only a portion of the table is shown here for guidance regarding its form and content. The full table is available in the online version of the journal (see Supporting Information and Appendix A). The columns are labelled as follows: column 1: dd/mm/20yy; column 2: normalized JD = JD - 245 0000; column 3: value of the sky background (ADU); column 4: number of combined frames for the given observing day; columns 5-6: full width at half-maximum (FWHM) of the seeing disc in arcsecond and signalnoise-ratio measured for the star R1; columns 7-10: relative magnitude of the A, B, C, D lensed components to the R2 reference star (mag) and their photometric error bars. The method used for calculating the error bars is presented in Appendix B; column 11: Relative magnitude of the reference star R2 to R3 (mag) and its photometric error bars, which were obtained via aperture photometry using the DAOPHOT/IRAF Software.

\begin{tabular}{|c|c|c|c|c|c|c|c|c|c|c|}
\hline Civil date & $\mathrm{JD}$ & $S_{\mathrm{bg}}$ & $N$ & FWHM & SNR & A & B & $\mathrm{C}$ & $\mathrm{D}$ & R2 \\
\hline 1 & 2 & 3 & 4 & 5 & 6 & $\begin{array}{c}7 \\
R \text { band }\end{array}$ & 8 & 9 & 10 & 11 \\
\hline- & - & - & - & - & - & - & - & - & - & - \\
\hline $05 / 05 / 03$ & 2765 & 243 & 4 & 1.00 & 113 & $1.121 \pm 0.010$ & $1.370 \pm 0.014$ & $1.486 \pm 0.013$ & $1.698 \pm 0.016$ & $-0.104 \pm 0.002$ \\
\hline 07/05/03 & 2767 & 284 & 4 & 0.79 & 109 & $1.150 \pm 0.006$ & $1.342 \pm 0.007$ & $1.504 \pm 0.007$ & $1.677 \pm 0.009$ & $-0.129 \pm 0.001$ \\
\hline- & - & - & - & - & - & $\begin{array}{c}- \\
V \text { band }\end{array}$ & - & - & - & - \\
\hline- & - & - & - & - & - & - & - & - & - & - \\
\hline 07/05/03 & 2767 & 136 & 4 & 0.86 & 77 & $1.047 \pm 0.035$ & $1.308 \pm 0.116$ & $1.385 \pm 0.031$ & $1.591 \pm 0.077$ & $0.035 \pm 0.011$ \\
\hline $29 / 05 / 03$ & 2789 & 143 & 4 & 1.13 & 66 & $1.043 \pm 0.023$ & $1.367 \pm 0.031$ & $1.346 \pm 0.027$ & $1.735 \pm 0.038$ & $0.034 \pm 0.013$ \\
\hline - & - & - & - & - & - & - & - & - & - & - \\
\hline
\end{tabular}

have a first known set of $\operatorname{PSF}(i, j)$, we have then to minimize the $\chi^{2}$ in order to determine the intensities $I_{k}$. It can be done by equating the partial derivatives $\partial \chi^{2} / \partial I_{l}$ to zero:

$$
\begin{aligned}
\frac{1}{2} \frac{\partial \chi^{2}}{\partial I_{l}}= & \sum_{i, j=1}^{N, M}\left[\operatorname{PSF}\left(i-i_{l}, j-j_{l}\right)\right. \\
& \left.\times \frac{\sum_{k=1}^{K} I_{k} \cdot \operatorname{PSF}\left(i-i_{k}, j-j_{k}\right)-D(i, j)}{\sigma^{2}(i, j)}\right]=0 .
\end{aligned}
$$

This relation can be simplified as:

$$
\begin{array}{r}
\sum_{k=1}^{K} \sum_{i, j=1}^{N, M} \frac{\operatorname{PSF}\left(i-i_{l}, j-j_{l}\right) \cdot \operatorname{PSF}\left(i-i_{k}, j-j_{k}\right)}{\sigma^{2}(i, j)} \cdot I_{k} \\
=\sum_{i, j=1}^{N, M} \frac{D(i, j) \cdot \operatorname{PSF}\left(i-i_{l}, j-j_{l}\right)}{\sigma^{2}(i, j)},
\end{array}
$$

which leads to

$\sum_{k=1}^{K} M_{l, k} \cdot I_{k}=C_{l}$,

with

$M_{l, k}=\sum_{i, j=1}^{N, M} \frac{\operatorname{PSF}\left(i-i_{l}, j-j_{l}\right) \cdot \operatorname{PSF}\left(i-i_{k}, j-j_{k}\right)}{\sigma^{2}(i, j)}$

and

$C_{l}=\sum_{i, j=1}^{N, M} D(i, j) \cdot \frac{\operatorname{PSF}\left(i-i_{l}, j-j_{l}\right)}{\sigma^{2}(i, j)}$

Equation (5) can be rewritten in a vectorial form:

$M \cdot \vec{I}=\vec{C} \quad$ or $\quad \vec{I}=M^{-1} \vec{C}$,

and it is easy to derive the components of the vector $\vec{I}\left(I_{1}, \ldots, I_{k}\right)$ if we assume that we know what are the values of the PSF. We thus proceed in an iterative way.

Suppose that the PSF, centred on $(0,0)$ covers $I \times J$ pixels, where $I=2 M_{I}+1$ and $J=2 M_{J}+1$. In the remainder, we use two different coordinate systems in order to locate one pixel on such a grid: $i^{\prime} \in$ $\left[-M_{I}, M_{I}\right], j^{\prime} \in\left[-M_{J}, M_{J}\right]$ and the pixel number $n=i^{\prime}+\left(j^{\prime}-\right.$ $1)\left(2 M_{I}+1\right) \in[1, I \times J]$. So, for a given pair of coordinates $\left(i^{\prime}, j^{\prime}\right)$ we obtain a unique value of $n$, and vice versa.
Considering any value for the number $n \in[1, I \times J]$ specific to a given PSF element, we know that only $K$ values of $D(i, j)$ will be concerned over the whole frame. As written before, a value of $n$ defines a pair of values $\left(i^{\prime}, j^{\prime}\right)$. Knowing that there is a number $K$ of PSFs, we should consider each of these independently. Therefore, for a value of $l \in[1, K]$ and a given pair of coordinates $\left(i^{\prime}, j^{\prime}\right)$, it is straightforward to find the absolute value $\left(i_{n l}, j_{n l}\right)$ of the position of the PSF $(1)$ in the frame. If $\left(i_{l}, j_{l}\right)$ represent the absolute central position of the $\operatorname{PSF}(1)$, then $i_{n l}=i_{l}+i^{\prime}$ and $j_{n l}=j_{l}+j^{\prime}$.

We can now perform the derivation of the $\chi^{2}$ function given in equation (2) with respect to the chosen $\operatorname{PSF}(n)$. We find

$$
\begin{aligned}
\frac{\partial \chi^{2}}{\partial \operatorname{PSF}(n)}= & 2 \sum_{l=1}^{K}\left[\frac{\sum_{k \neq l}^{K} I_{k} \cdot \operatorname{PSF}\left(i_{n l}-i_{k}, j_{n k}-j_{k}\right)}{\sigma^{2}\left(i_{n l}, j_{n l}\right)}\right. \\
& \left.+\frac{I_{l} \cdot \operatorname{PSF}(n)-D\left(i_{n l}, j_{n l}\right)}{\sigma^{2}\left(i_{n l}, j_{n l}\right)}\right] \cdot I_{l}=0
\end{aligned}
$$

and thus,

$$
\begin{aligned}
& \operatorname{PSF}(n) \cdot \sum_{l=1}^{K} \frac{I_{l}^{2}}{\sigma^{2}\left(i_{n l}, j_{n l}\right)} \\
& =-\sum_{l=1}^{K}\left[\frac{\sum_{k \neq l}^{K} I_{k} \cdot \operatorname{PSF}\left(i_{n l}-i_{k}, j_{n l}-j_{k}\right)-D\left(i_{n l}, j_{n l}\right)}{\sigma^{2}\left(i_{n l}, j_{n l}\right)}\right] \cdot I_{l} .
\end{aligned}
$$

The updated value of $\operatorname{PSF}(n)=\operatorname{PSF}\left(i^{\prime}, j^{\prime}\right)$ can be recalculated as

$$
=\frac{\sum_{l=1}^{K}\left[\frac{D\left(i_{n l}, j_{n l}\right)-\sum_{k \neq l}^{K} I_{k} \cdot \operatorname{PSF}\left(i_{n l}-i_{k}, j_{n l}-j_{k}\right)}{\sigma^{2}\left(i_{n l}, j_{n l}\right)}\right] \cdot I_{l}}{\sum_{l=1}^{K} \frac{I_{l}^{2}}{\sigma^{2}\left(i_{n l}, j_{n l}\right)}}
$$

with the new intensities $I_{l}$ being solutions of equation (8).

At the first iteration, the input point spread function $\operatorname{PSF}\left(i-i_{k}\right.$, $j-j_{k}$ ) can be any peaked 2D function or the image of some nearby reference star. It is then used as the input $\operatorname{PSF}(n)$ in equation (8) to derive a set of new central intensities. The latter are then injected 


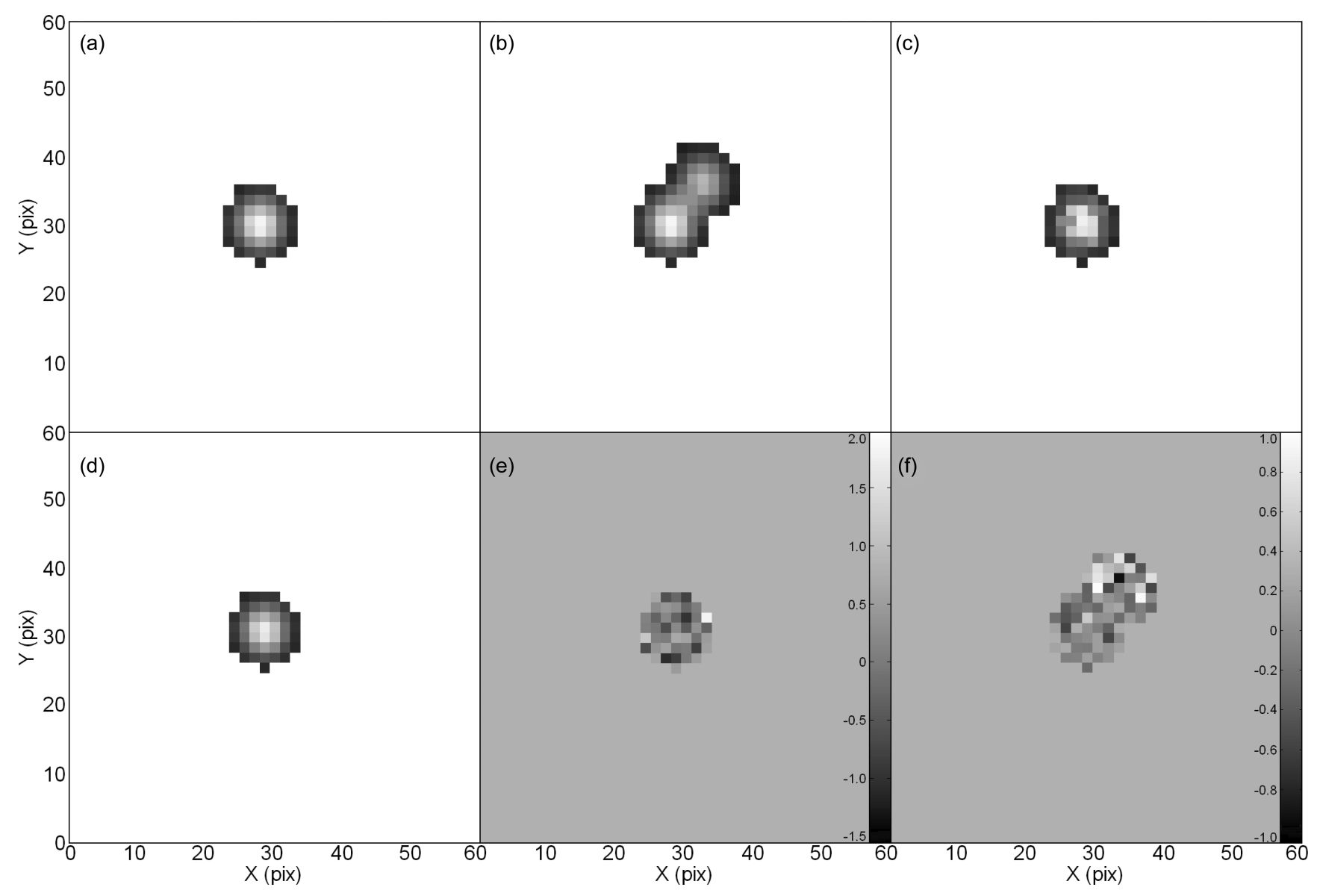

Figure 3. (a) Real PSF; (b) composed image with two real PSFs including Poisson noise; (c) initial PSF; (d) derived PSF after a series of iterations; (e) residuals between the derived and the real PSF, normalized to one standard deviation $(1 \sigma)$ of the expected Poisson noise; (f) residuals after subtraction of a double PSF, also normalized to $1 \sigma$.

in equation (11) to derive new values of $\operatorname{PSF}(n)$ and so on. After a series of iterations, we derive the best values of $\operatorname{PSF}(n)$ and of the central intensities which minimize the $\chi^{2}$.

As we see, the two equations (8) and (11) constitute the core of the adaptive PSF-fitting method. By using them alternatively and starting from a first estimation of the PSF, the central intensities $I_{k}$ can be numerically calculated, as well as the PSF. The adaptive PSFfitting method has a major advantage over the classical PSF-fitting method: there is no need to make use of any assumption concerning the invariance of the PSF over the CCD frame. This calculated PSF is determined from the lensed components themselves, not from a reference star. We have performed the tests with different shapes of the initial PSF and found that the result does not depend on it. As the iterations proceed, the PSF always converges towards a stable final shape which is typical for a given observation. Indeed, in equation (1) we only made the assumption that the PSFs of each component are identical to each other. This assumption of the local frame invariance is only effective over the distance scale of the gravitational lens system. This approximation is much less restrictive than the global frame invariance assumed in the classical PSF-fitting method.

The only requirements specific to the adaptive PSF-fitting method are:

(i) the positions of each component on the CCD frame have to be accurately known, at least within a pixel size precision; (ii) the CCD frames must have a good signal-to-noise ratio. It is then possible in our case to pinpoint the four overlapping components of $\mathrm{H} 1413+117$.

Let us note that these requirements are shared by most of photometric techniques dedicated to the flux measurement of very nearby objects. Thus, the absence of additional assumptions implies that the adaptive PSF-fitting photometric method can be applied to a larger range of observations than any other photometric technique.

We tested our method using artificially generated images. First of all, we chose some 2D PSF (see Fig. 3a). Secondly, we composed a CCD frame which is the sum of two real PSFs with a flux ratio and a separation between their centres equal to 0.7 and 5 px (pixels), respectively. Moreover, we added random Poisson noise to this frame (Fig. 3b). For the first step of the adaptive PSF fitting, we chose some initial PSF to solve equations (5)-(8) (Fig. 3c). We carried out a series of iterations until the total $\chi^{2}$ reached a given minimum stable value. As a result, we derived a PSF (Fig. 3d) which is very similar to the real one. The residuals between the derived and the real PSF are shown in Fig. 3(e) after normalization to $\sigma(i$, $j$ ), one standard deviation of the expected Poisson noise. As can be seen, there is no significant difference between the two, thus we have correctly recovered the primary, real PSF.

Next, we checked the results of the method for different flux ratios and distances between the centres of the components. First, we fixed the separation between the two components to Dist $=5 \mathrm{px}$ and applied the adaptive PSF-fitting method for three different 

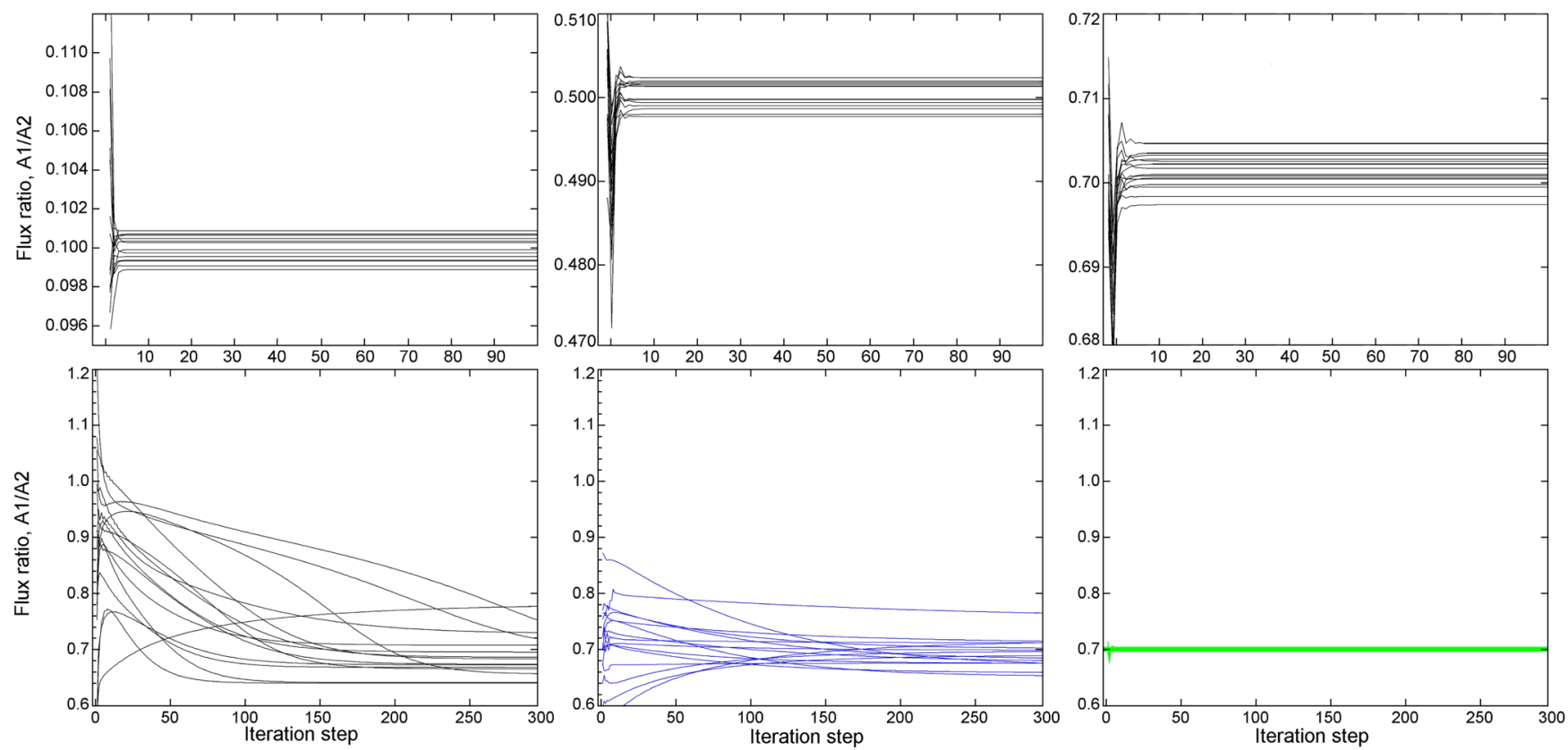

Figure 4. Examples of the flux ratio and $\chi^{2}$ value evolutions as a function of the number of iterations for different preset flux ratios and separations. Top row: the separation between the two components is Dist $=5$ px everywhere, the flux ratios $A_{1} / A_{2}=0.1,0.5,0.7$. Bottom row: flux ratios $A_{1} / A_{2}=0.7$ everywhere, the separations are $D=1,3,5 \mathrm{px}$.

values of the flux ratio $A_{1} / A_{2}=0.1,0.5,0.7$. For each fixed value of the flux ratio and distance, we randomly generated 15 frames affected by Poisson noise (Fig. 3b), and then applied to each of these the adaptive PSF fitting. Fig. 4 (top row) shows the evolution of the flux ratios between the components as a function of the iteration step for different values of the flux ratio $A_{1} / A_{2}$. As can be seen, the curves converge towards the adopted value after just a few iterations. Average values of the flux ratios are $0.100 \pm 0.001,0.500$ $\pm 0.002,0.7 \pm 0.003$, respectively. Secondly, we fixed the flux ratio between the two components to 0.7 and applied the adaptive PSF-fitting method for three different values of their separation: 1, 2, 5 px. Obviously, wider is the pair, better is the final accuracy. The bottom row of Fig. 4 shows how the flux ratio converges as a function of the number of iterations. Only small changes occur after the 10 first iterations, with the calculated flux ratios: $0.730 \pm$ $0.091,0.720 \pm 0.075,0.700 \pm 0.002$.

\section{PHOTOMETRY OF THE FOUR LENSED COMPONENTS OF H1413+117}

We shall now describe the application of the adaptive PSF-fitting photometric method to the case of the Clover Leaf. Since most of our CCD frames were obtained in the short-focus mode, we shall focus our attention to the problems encountered with this type of CCD frames.

Our method does not require a high accuracy on the lensed component positions - it is enough to know them within a single pixel size precision. To determine the relative distances between the components we used all available information about the coordinates of the lensed images. We adopted the astrometric measurements provided by Turnshek et al. (1997), Chantry \& Magain (2007) and MacLeod et al. (2009) as a first approximation to fix the component positions which were registered on our detector. Inaccuracies in the positions of the components were taken into account when estimating the photometric error bars (see Appendix B).
However, application of the adaptive PSF-fitting photometric method to the original frames did not give any satisfactory results, first of all, due to the small signal-to-noise ratio. On the other hand, $\mathrm{H} 1413+117$ is a multiple object with small angular separations between the lensed components. The combination of these two factors did not allow us to distinguish (even under good seeing conditions) the separate peaks of the lensed images. In this case, the adaptive PSF-fitting photometric method led to non-physical results - negative fluxes or implausible flux ratios of the components. In general, the mathematical model of the adaptive PSF-fitting method allows us to reach such solutions. To avoid such difficulties, we have decided to artificially increase the resolution of the images using a bi-cubic interpolation of the original frames (Press et al. 1992). The details of the interpolation are explained in Ricci et al. (2011) and were also applied by Ricci et al. (2013) and Elyiv et al. (2013).

An average sky background value is simply subtracted before applying the adaptive PSF-fitting photometric method (this is not implicitly included in equations (8) and (11)).

A typical result calculated by means of the adaptive PSF-fitting photometric method on to a bi-cubic interpolated CCD frame is shown in Fig. 5. Here are the original frame (a) and a frame which was pixelated 10 times (b), which means that each pixel of the original CCD frame was bi-cubic interpolated from the 10 inner points equidistant from one another. The frame (c) illustrates the resulting adaptive PSF photometric model; and in (d) the residuals between the real and modelled frames, normalized to the one sigma standard noise deviation $\sigma(i, j)$ image frame. The residual frames show good general self-consistency; their individual values are in the range of $\pm(2-3) \sigma(i, j)$. The criterion used for stopping the iteration was to reach a stable convergence for the flux ratios of the lensed components. In the case of good seeing conditions, about 1200-1600 iterations were needed to reach reliable results.

For all images of the Clover Leaf, we used the image of the star R1 as the initial PSF, observed on 2004 May 09 under good conditions. 

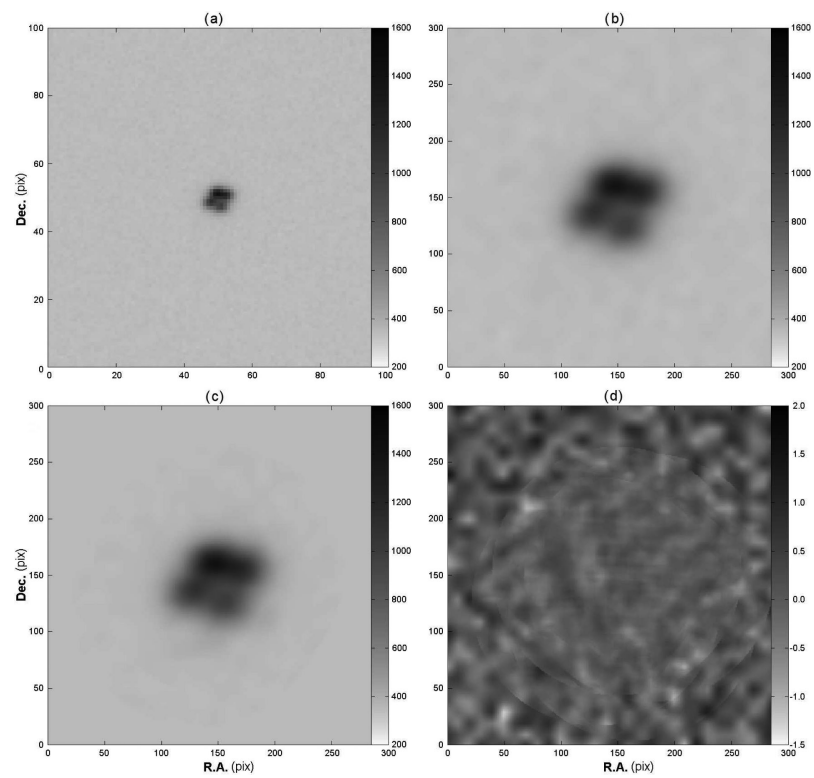

Figure 5. Results of the application of the adaptive PSF-fitting photometric method to our CCD frames. (a) The original frame, obtained in the $R$ band on 2003 February 10 (the same as in Fig. 2 at right); (b) 10-times pixelated and bi-cubic interpolated version of the original frame; (c) the resulting adaptive PSF-fitting photometric model of H1413+117; (d) the residual image between the real frame and the simulated one. This frame is given in units of the standard noise deviation $\sigma(i, j)$. The presented scale and overall distribution of the residuals are characteristic of all the considered CCD frames.

\section{LIGHT CURVES AND PHOTOMETRIC UNCERTAINTIES}

At first, we would like to present the instrumental photometric light curves of $\mathrm{H} 1413+117$ as a whole and those of the reference star R2 in the $V$ and the $R$ bands, as well as the $V-R$ colour curves (see Fig. 6). The light curves were obtained via aperture photometry using the DAOPHOT/IRAF software. The light curves of R2 are displayed relatively to the star R3 (see Fig. 1) and are shifted down by 0.25 mag for clarity. Given the apparent photometric stability of the star R2 with respect to the star R3, we have decided to use hereafter the star R2 as a reference star. The relative light curve R1-R2 (see Figs 7 and 8) is also flat and stable, but has greater dispersion [this is due to the fact that the magnitude difference between R1 and R2 (or R3) is around $2 \mathrm{mag}$ ].

As seen in Figs 6-8, the quasar displays some active variability with an amplitude $\approx 0.15$ mag between the end of the season 2003 and the middle of 2005 (the brightness of the D-lensed component reached its maximum at this moment and then diminished smoothly during the following years). The mentioned activity between the seasons 2003 and 2005 does not show up similarly in the two photometric bands. As we can see, there exists a brightness-colour dependence: a decrease in the brightness of the quasar leads to a reddening, and vice versa. This is an interesting observation and might possibly be explained as due to micro-lensing (as for the case of Q2237+0305, Wambsganss \& Paczynski 1991) and/or as internal processes in the quasar itself (as in UM673, Koptelova et al. 2012; Ricci et al. 2013). To assert this statement, we must separately analyse the light curves and the colour curves of the lensed images.

We have applied our adaptive PSF-fitting method to the lensed images of $\mathrm{H} 1413+117$ to derive their appropriate flux measure-
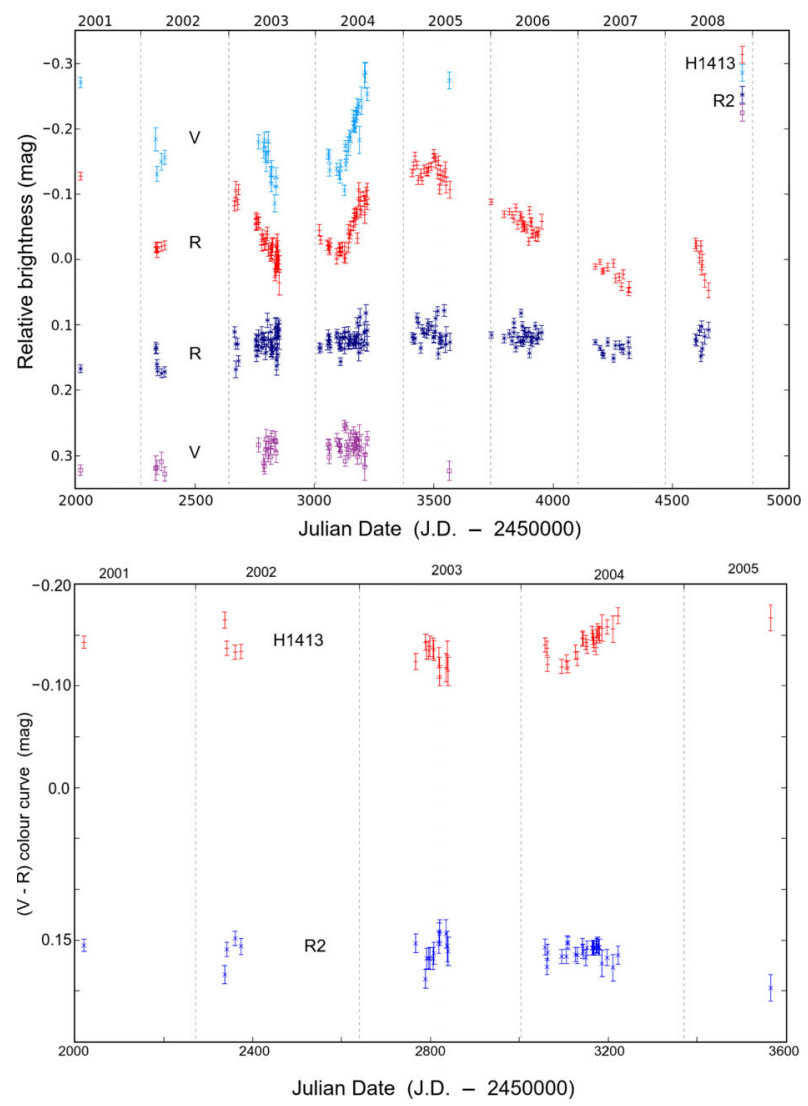

Figure 6. Top: light curves of the quasar $\mathrm{H} 1413+117$ (blue and red points) and of the reference star R2 (violet and dark-blue) in the $V$ - and $R$-pass band, respectively. For clarity, both light curves of R2 are shifted down by 0.25 mag. Bottom: $V-R$ colour curves of the quasar and of the reference star R2. Quasar light curves are plotted relative to the reference star R2, and R2 are plotted relative to the star $\mathrm{R} 3$.

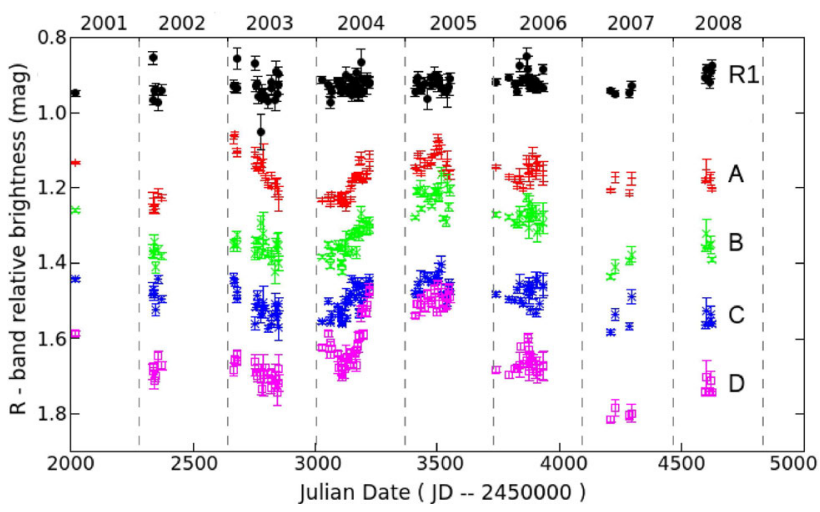

Figure 7. $R$-band light curves for the $\mathrm{A}, \mathrm{B}, \mathrm{C}, \mathrm{D}$ lensed components of H1413+117 and of the reference star R1, which has been shifted up by $-0.5 \mathrm{mag}$ for clarity, with respect to the reference star R2. The years which correspond to the observing seasons are indicated at the top.

ments. The next task was then to estimate the photometric error bars affecting each point of the light curves. To calculate these photometric error bars, we used Monte Carlo simulations of our CCD frames including the different sources of noise such as modelling of the Clover Leaf CCD frames, the GLS signal, the sky background and read-out noise, flat-field variation, random positional centring (the details of this procedure are given in Appendix B). 


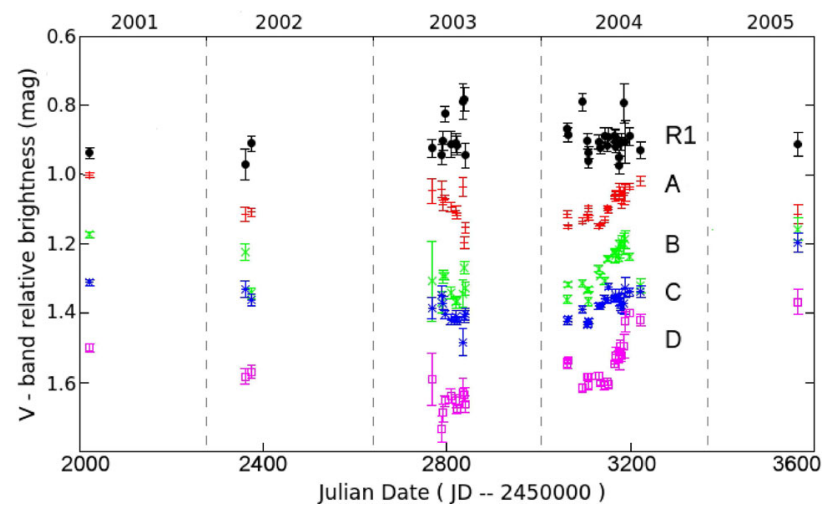

Figure 8. $V$-band light curves for the A, B, C, D lensed components of H1413+117 and of the reference star R1, which has been shifted up by -0.75 mag for clarity, with respect to the reference star R2. The years which correspond to the observing seasons are indicated at the top.

For each day of observation, we generated 500 simulated CCD frames and applied the developed procedure of adaptive PSF fitting to each of them. The calculated fluxes and consequently, the lensed component's magnitudes displayed a Gaussian distribution. For this reason, the photometric error bars associated with each observing night are defined as the standard deviations of the magnitudes out of the 500 corresponding simulations.

In our analysis, we ignored the presence of the gravitational lens near the centre of the system because it is too faint to be detected on our CCD frames (Chantry \& Magain 2007).

The $R$-band and the $V$-band light curves of the lensed images of $\mathrm{H} 1413+117$ and the magnitude differences R1-R2 are presented in Figs 7 and 8 (see also Table 2). Note that the light curves of the lensed images are plotted relatively to the star R2. These curves clearly show that the fluxes of the H1413+117 lensed components are varying continuously and are correlated with the overall brightness change of the quasar (see Fig. 6). A detailed analysis of the light curves will be given after estimating the time delays, as well as a discussion of the colour variations.

\section{TIME DELAY CALCULATIONS}

The time delays $(\Delta t)$ constitute an important feature of gravitational lens systems (Refsdal 1964a,b). These quantities are directly related to the gravitational potential of the lensing galaxy and to the geometrical properties of the Universe at large distances. A wide variety of algorithms have been developed for the determination of time delays in gravitational lens systems. The methods presented here are based on the chi-squared $\chi^{2}$ (Gaynullina et al. 2005; Ullán et al. 2006) and dispersion $D^{2}$ (Pelt et al. 1996) minimizations. In general, all the techniques proceed in a similar way - they try to find how to match at best two given light curves. Usually, the chi-squared method is used in the case of well-sampled light curves with different types of interpolation between the inner points (Kundic et al. 1997). In the case of poorly sampled data, Gil-Merino, Wisotzki \& Wambsganss (2002) advise to use the dispersion methods such as the Pelt's algorithms (Pelt et al. 1996). These two techniques $\left(\chi^{2}\right.$ and $D^{2}$ ) were also used by Goicoechea $\&$ Shalyapin (2010) to determine the time delay values between three pairs of the components of $\mathrm{H} 1413+177$ in 2008 . We want to compare the results provided by the two approaches when applied to a moderately sampled data set like ours. The chi-squared $\chi^{2}$ method that we used is well described in Gaynullina et al. (2005). But in our case, we considered three

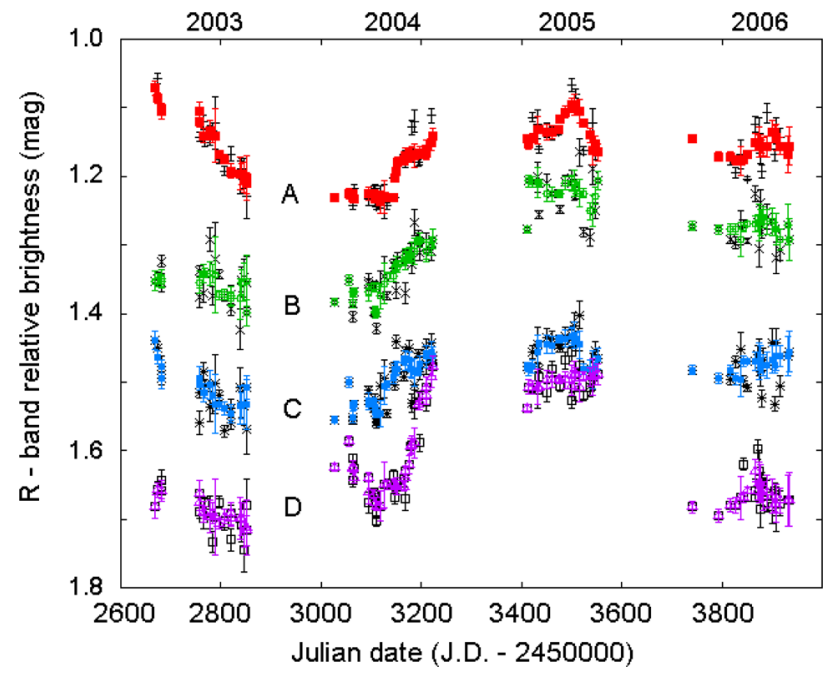

Figure 9. Illustration of the five-point median filtering of the light curves during the 2003-2006 seasons. The background black points are the same as in Fig. 7.

values of the maximum gap ( $\delta=20,25$ and $30 \mathrm{~d}$ ), within which linear interpolation was allowed. Furthermore, we have tested the dispersion method using two statistical values: $D_{2}^{2}$ and $D_{3}^{2}$, which correspond to the formulas (7) and (8) of the paper by Pelt et al. (1996). Here, we have used the same $\delta=20,25$ and $30 \mathrm{~d}$ as the maximum distance between two observations which can be considered as sufficiently near.

We have considered the six possible pairs of light curves: $A B$, $\mathrm{AC}, \mathrm{AD}, \mathrm{BC}, \mathrm{BD}$ and $\mathrm{CD}$. As we can see on the plots (Figs 68 ), the brightness of the components varies with a high amplitude (up to $0.2 \mathrm{mag}$ ) over long time-scales. But the very long (several months) gaps between the seasons do not allow us to use the whole light curves. Because of unpredictable intrinsic and possible microlensing variations in the light curves of the components, we could not carry out any interpolation within these interseason gaps.

We thus decided to work with the individual fragments of the $R$-band light curves that correspond to the four observing seasons between 2003 and 2006. These seasons contain a sufficient number of data points (32 in 2004 and 20 in the others) to allow a relevant analysis. Next, we decided to apply a smoothing procedure to the light curves. We used a five-point median sliding filter, which corresponds, on average, to a 20 -d filtering window. This allowed us to reduce the scattering in the photometry, i.e. to damp any sudden change in the light curves caused, first of all, by the conditions of observation and possible secondary fluctuations of the quasarsource, induced quasar micro-lensing effects, etc. (Fig. 9). This type of light curves is referred to as MFLC (median filtered light curves), contrarily to DLC (direct light curves), for which we did not use any filtering. We used both types of light curves separately for the calculation of the $\Delta t$ values.

To provide an estimation of the uncertainties and confidence level of the derived time delays, we adopted, as usually, a Monte Carlo method, i.e. we generated a large number of synthetic light curves and applied to each of them, the same calculation procedure to determine the corresponding time delay $\Delta t$. We used two different procedures to generate these artificial light curves:

(i) Our first procedure has consisted in creating synthetic light curves by simply adding a random number to the magnitude of each point of the real light curve. This number follows a normal 
Table 3. Estimates of the time delays (in days) obtained for the year 2004 as a function of the methods applied and the type of light curves analysed for three values of $\delta=20,25$ and $30 \mathrm{~d}$. Only time delay values derived on the basis of light curves of the group I are shown here. DLC is direct simulated light curves, MFLC is median filtered light curves. If the histogram in the distribution of time delays does not allow to fix a peak, we mark it as Indefinite value (INDEF). 'no' or 'yes' are answers to the question of the first and second steps of the self-consistency test. In our $\Delta t$ calculations, we assumed that: $\Delta t_{\mathrm{XY}}=t_{\mathrm{Y}}-t_{\mathrm{X}}$.

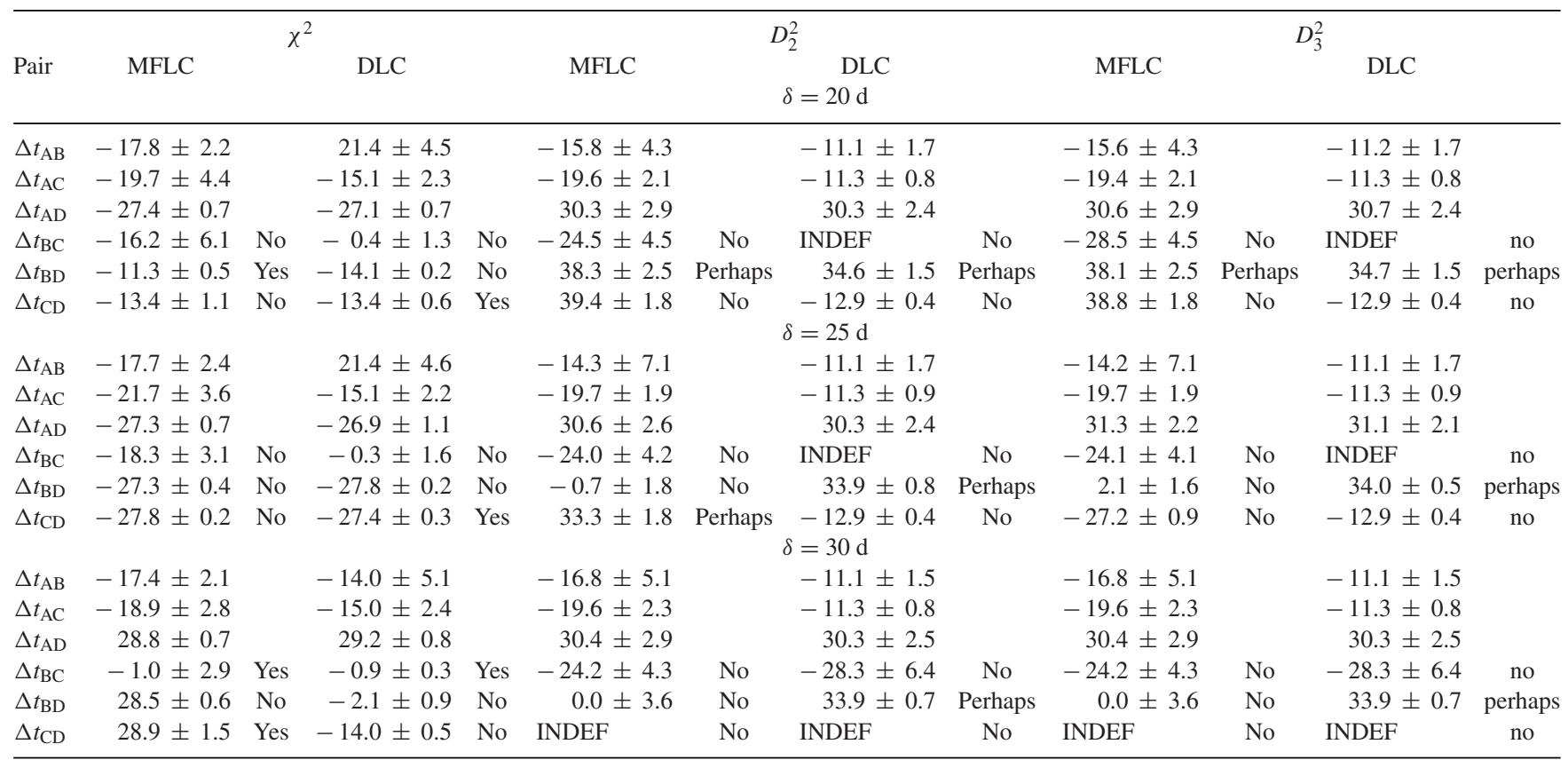

distribution, centred on zero, with an FWHM equal to the photometric error bar of the point. This forms the group I of light curves.

(ii) We now describe the second procedure. Since we have previously calculated random magnitudes in accordance with a Gaussian distribution to estimate the photometric uncertainties, we have used these same data to re-create a new set of simulated light curves. The photometric error bars of each point of the light curves is the standard deviation of the set of the 500 random magnitudes corresponding to this point (see Section 5). Thus, we have generated another set of synthetic light curves by randomly choosing one of these calculated magnitudes and declared it as a point of the simulated light curve. To our opinion, this procedure of simulated light-curve generation is more reliable because it perfectly respects the magnitude distributions given by our adaptive PSF-fitting method: if the distribution is not perfectly normal, this fact will automatically be taken into account in the simulated light curves. This forms the group II of light curves.

We have compared the predictions following the analysis of these two sets of light curves. For each of these sets, we have generated 10000 synthetic light curves. We have carried out $\Delta t$ calculations covering a range of $[-40,40] \mathrm{d}$ (and $[-60,20] \mathrm{d}$ for the pairs BD and $\mathrm{CD}$ ) with a step of $0.2 \mathrm{~d}$, and values of magnitude differences between the components in the pairs covering appropriate ranges with a step of $0.002 \mathrm{mag}$.

As a result, we got a large number of independent and different (and contradicting, in many cases) values of time delays. These represent the average values of the main peaks in the $\Delta t$ histograms. Note that we have obtained several hundred possible predictions of $\Delta t: 4 \mathrm{yr}(2003-2006) \times 6$ pairs $(\mathrm{AB}, \mathrm{AC}, \mathrm{AD}, \mathrm{BC}, \mathrm{BD}$ and $\mathrm{CD}) \times$ 3 formulas $\left(\chi^{2}, D_{2}^{2}, D_{3}^{2}\right) \times 2$ groups of simulated light curves (the set generated using normal distributions and the set generated using random choice) $\times 2$ types of light curves (DLC and MFLC) $\times 3$ values of $\delta$. Preliminary analysis of the time delay values showed that the observational season 2004 gives the most relevant results. See Table 3, where we have shown the only results of the group I (the group II gives almost the same results). In Fig. 9, we see that the A, B and C components varied in parallel in 2004, while D had a 'peculiar' behaviour probably induced by micro-lensing. However, we also found indirect evidence of micro-lensing in the component $\mathrm{B}$ on the basis of the colour index $V-R$ (see Section 7). But since this variation is not evident in the corresponding light curve, we have considered that the $R$-band light curve of the $\mathrm{B}$ component is clearly similar to those of $A$ and $C$.

We now have thirty-six sets of solutions: according to the used formulas $\left(\chi^{2}, D_{2}^{2}, D_{3}^{2}\right)$, three values of $\delta$, two types of light curves (MFLC and DLC) and two groups of simulated light curves. To determine which of these time delay values are the most appropriate, we have adopted the main following criterion: a correct prediction must be self-consistent. We decided that a self-consistency test should be structured in two steps.

In the first step, we have to answer the question - are the $\Delta t_{\mathrm{AB}}$, $\Delta t_{\mathrm{AC}}$ and $\Delta t_{\mathrm{BC}}$ consistent with each other?, i.e.

$\Delta t_{\mathrm{BC}} \cong \Delta t_{\mathrm{AC}}-\Delta t_{\mathrm{AB}}$

In the second step of the self-consistency test, we look whether the $\Delta t_{\mathrm{AB}}, \Delta t_{\mathrm{AC}}, \Delta t_{\mathrm{AD}}, \Delta t_{\mathrm{BC}}, \Delta t_{\mathrm{BD}}$ and $\Delta t_{\mathrm{CD}}$ time delays are consistent with each other?, i.e.

$\Delta t_{\mathrm{BD}} \cong \Delta t_{\mathrm{AD}}-\Delta t_{\mathrm{AB}}$
$\Delta t_{\mathrm{CD}} \cong \Delta t_{\mathrm{BD}}-\Delta t_{\mathrm{BC}}$

In the first step, the answer 'yes' is mandatory. However, in the second step, we may get some 'anomaly' in $\Delta t_{\mathrm{BD}}$ and/or $\Delta t_{\mathrm{CD}}$ (difference between the estimated value and the expected one). These anomalies may be due to micro-lensing, and simply indicate that delays involving the light curve of $D$ are not reliable (the formal errors do not incorporate a main systematic: micro-lensing). 
Table 4. Final estimates of the time delays $\Delta t$ (in days) and magnitude differences $\Delta m$ (in mag) on the basis of the $\chi^{2} / \mathrm{MFLC} / \delta=30 \mathrm{~d} /$ approach with estimates of the measurement accuracy. On the left, results for the group I synthetic light curves are given. On the right, the same but for group II. Here the B and C images are leading, then comes A, and at last, the D component is trailing the most.

\begin{tabular}{lrc|rc}
\hline & \multicolumn{2}{c}{ Group I of light curves } & \multicolumn{2}{c}{ Group II of light curves } \\
Pair & \multicolumn{1}{c}{$\Delta t$} & $\Delta m$ & \multicolumn{1}{c}{$\Delta t$} & \multicolumn{1}{c}{$\Delta m$} \\
\hline$\Delta t_{\mathrm{AB}}$ & $-17.4 \pm 2.1$ & $0.148 \pm 0.003$ & $-18.2 \pm 2.0$ & $0.149 \pm 0.004$ \\
$\Delta t_{\mathrm{AC}}$ & $-18.9 \pm 2.8$ & $0.309 \pm 0.004$ & $-18.1 \pm 2.5$ & $0.309 \pm 0.004$ \\
$\Delta t_{\mathrm{AD}}$ & $28.8 \pm 0.7$ & $0.414 \pm 0.003$ & $28.6 \pm 0.6$ & $0.415 \pm 0.003$ \\
$\Delta t_{\mathrm{BC}}$ & $-1.0 \pm 2.9$ & $0.159 \pm 0.004$ & $-0.5 \pm 2.7$ & $0.159 \pm 0.004$ \\
$\Delta t_{\mathrm{BD}}$ & $28.5 \pm 0.6$ & $0.277 \pm 0.003$ & $-0.4 \pm 1.3$ & $0.279 \pm 0.004$ \\
$\Delta t_{\mathrm{CD}}$ & $28.9 \pm 1.5$ & $0.115 \pm 0.003$ & $28.6 \pm 0.8$ & $0.115 \pm 0.004$ \\
\hline
\end{tabular}

A comparison between the measured and expected time delays for the $B C, B D$ and $C D$ pairs is shown in Table 3 (see the answers 'yes' or 'no'). These results suggest that the $\chi^{2} / \mathrm{MFLC} / \delta=30$ /group I approach works much better than the other ones. Therefore, we have adopted the latter set as the final time delays (see Table 4). However, it should again be noted that the uncertainty in the $\Delta t_{\mathrm{AD}}$, $\Delta t_{\mathrm{BD}}$ and $\Delta t_{\mathrm{CD}}$ time delays is likely underestimated, because when calculating those time delays we did not take into account the effect of micro-lensing.

The change in the flux of the light curves that occurred at about 3150JD allowed us to derive self-consistent values for the time delays. During these days, the brightness of the components increased by $\sim 0.06$ mag during about 1 month with a rate of $1.5 \times$ $10^{-3} \mathrm{mag} \mathrm{d}^{-1}$ (see Fig. 9), while the average rate in brightness variation for the individual season is about $6 \times 10^{-4} \mathrm{mag} \mathrm{d}^{-1}$. Thus, the predictions from the MFLC light curves (especially for the 2004 season) are more relevant than those of the direct light curves. Time delay and appropriate magnitude difference results are summarized in Table 4. According to the results of this table, we conclude that the proposed method for generating synthetic light curves (group II) works as well as the classic method and leads to quite similar results.

In Fig. 10, we have illustrated the combined light curve of H1413+117 corrected for the predicted time delays (Table 4, lefthand side) and magnitude shifts (the time delays obtained by the formula $\chi^{2}$ and $\delta=30 \mathrm{~d}$ on the basis of the group I light curves). Because of active variability of the components, the magnitude differences between the components are not constant, and vary from season to season. For example, in $2003 \Delta m_{\mathrm{AB}}=0.198, \Delta m_{\mathrm{AC}}=$ $0.364, \Delta m_{\mathrm{AD}}=0.557$; in $2004 \Delta m_{\mathrm{AB}}=0.148, \Delta m_{\mathrm{AC}}=0.309$, $\Delta m_{\mathrm{AD}}=0.414$; in $2005 \Delta m_{\mathrm{AB}}=0.103, \Delta m_{\mathrm{AC}}=0.328, \Delta m_{\mathrm{AD}}$ $=0.370$; in $2006 \Delta m_{\mathrm{AB}}=0.110, \Delta m_{\mathrm{AC}}=0.318, \Delta m_{\mathrm{AD}}=0.516$. These magnitude values correspond to the peaks in the magnitude shift distributions.

As can be seen from Table 4, the method of generating synthetic light curves based on randomly simulated magnitudes (see Section 5) has a right to exist, because this approach leads to quite comparable results as those derived by the classical method based on the normal distribution with a width equal to the photometric error bars.

\section{MICRO-LENSING AND COLOUR VARIATIONS}

The long-term light curves indicate the presence of micro-lensing effects, sometimes displaying a large amplitude with respect to
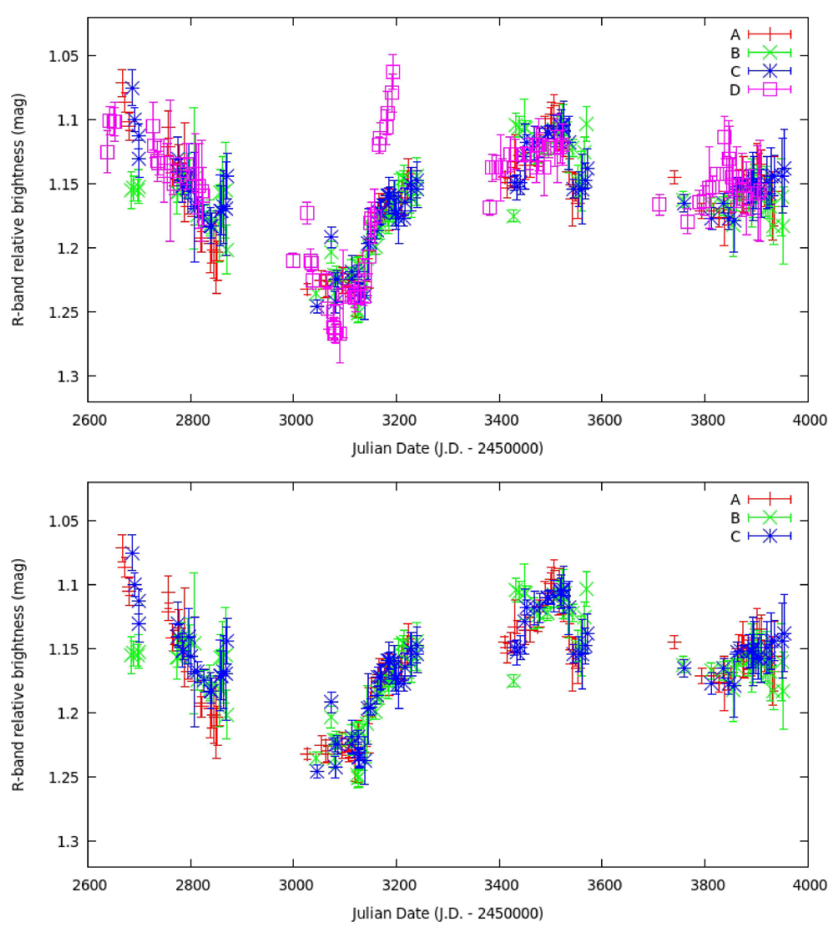

Figure 10. Combined light curve of $\mathrm{H} 1413+117$ for the MFLC case. These plots are made from the B, C, D (top) and B, C (bottom) light curves shifted in time and magnitude with respect to the $A$ component. The time delay values are those in Table 4 (left-hand side, MFLC, group I). But magnitude shifts between different seasons are different, probably due to micro-lensing effects (see text below).

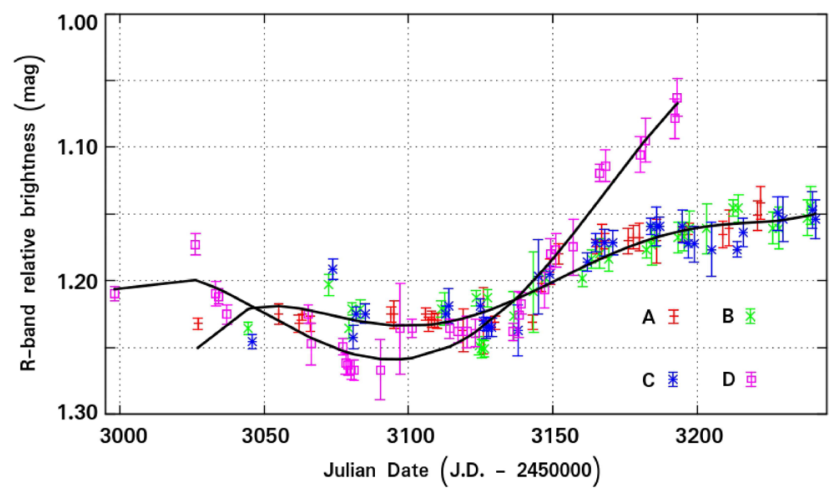

Figure 11. Combined light curves of H1413+117 during the 2004 season with appropriate $\Delta t$ and $\Delta m$ shifts and smoothed curves fitted with fifth order polynomials.

intrinsic variability. In Figs 7-9, we see that the maximum amplitude of the component variations is also of the order $\sim 0.15$ mag (see the A component between 2002 and the beginning of the 2003 season). The brightness variability of the components is undoubtedly due to a superposition of some intrinsic variability of the quasar and noncorrelated micro-lensing effects. Particularly noticeable changes are visible for the $D$ component, during the seasons 2004 and 2005: its brightness increased by $0.1 \mathrm{mag}$.

At the bottom of Fig. 11, the combined light curves for 2004 and their fifth order polynomial fits are presented. We fitted the A + B $+\mathrm{C}$ and $\mathrm{D}$ curves separately. The difference between those light curves is interpreted as the signature of a micro-lensing effect for the D component. In general, gravitational lens systems are affected 


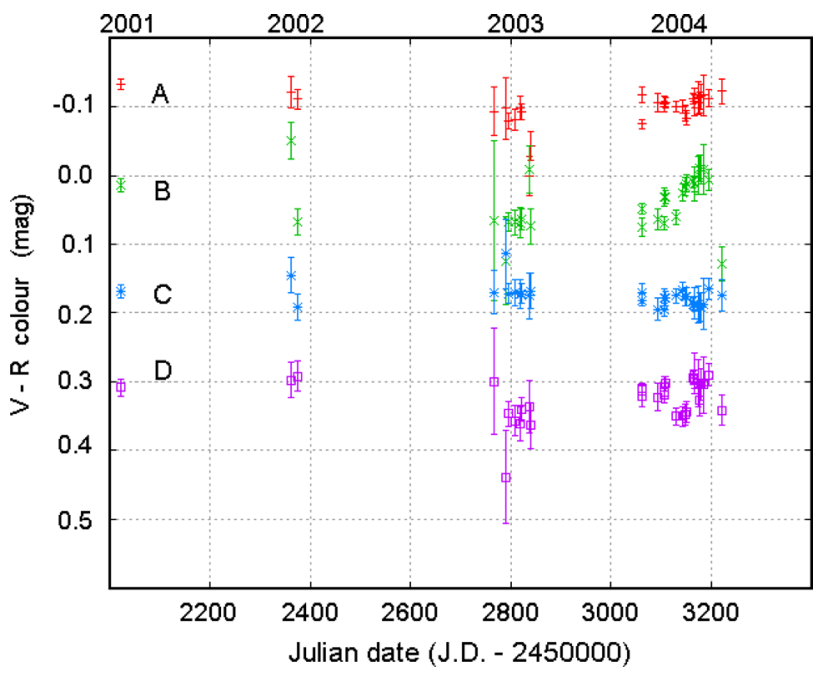

Figure 12. $V-R$ colour curves of the components. The colour values are near -0.1 mag. For clarity, the B, C and D colour curves have been shifted down by $0.1,0.3$ and $0.4 \mathrm{mag}$, respectively.

by micro-lensing with induced variability at the level of $10^{-4}$ mag d $^{-1}$ (Gaynullina et al. 2005; Vakulik et al. 2006; Goicoechea \& Shalyapin 2010; Tsvetkova et al. 2010). But here we found out one of the fastest micro-lensing effects with a rate of $10^{-3} \mathrm{mag} \mathrm{d}^{-1}$. As a consequence, the $\mathrm{D}$ component magnitude became almost comparable to that of the C component in 2005. In 2007, apparently, the induced micro-lensing flux increase stopped and the magnitude of the D component came back to its 'quasi-original' state.

The C component shows smaller light variations in 2005, while all the other components increased in brightness. It seems that its amplification decreased during this period. Furthermore, the magnitude change between 2005 and 2006 (as well as between 2006 and 2007) is smaller, contrary to the other components (except $D$ for the latter). Perhaps another amplifying micro-lensing event occurred at this time, during 2006 or 2007 , or it might be the same event, spreading over $3 \mathrm{yr}$.

The flux slope of the B component in 2003 is smaller than those of the other components. Perhaps it is due to a micro-lensing deamplification event that occurred before 2003 and ended sometime after our observations began, or a short micro-lensing amplification event occurring at the end of the observations for this year.

Another evidence of micro-lensing can be brought up after inspection of the colour light curves of the lensed components. Unusually brightness variations due to micro-lensing are chromatic, such as the intrinsic variations of the quasar. Therefore, colour curves have to show, after correction for the time delays, almost identical variations for each lensed component, except during micro-lensing events. And as we can see in Fig. 12, the colours of the B and D components were changing during 2004, becoming bluer, but not those of the other components. Brightness variation of the $\mathrm{B}$ component is larger in the $V$ band than in the $R$ band, which is also confirmed by the plot of the colour light curves (see Fig. 12). Their colour changes are unrelated because the slope of the B colour curve is clearly different from the slope shown by the D colour one. Furthermore, these colour curves confirm that the $\mathrm{B}$ component displayed a micro-lensing event as strong as the D-component event, although it was less visible in the light curves. Therefore, we confirm the presence of chromatic micro-lensing events in the Clover Leaf.

The $A$ component seems to be the most stable. Its light and colour behaviours during each season are similar to the behaviour of at least
Table 5. The lensed image (A-D) and deflector positions along with the mid-IR flux ratios for the H1413+117 system. The values of the lensed image positions come from Turnshek et al. (1997), while the deflector position comes from MacLeod et al. (2009).

\begin{tabular}{lccc}
\hline Component & $\begin{array}{c}\Delta \mathrm{RA} \\
\text { (in } \operatorname{arcsec})\end{array}$ & $\begin{array}{c}\Delta \mathrm{DEC} \\
\text { (in } \operatorname{arcsec})\end{array}$ & Mid-IR \\
\hline A & 0.0 & 0.0 & $\equiv 1.00$ \\
B & $0.744 \pm 0.003$ & $0.168 \pm 0.003$ & $0.84 \pm 0.07$ \\
C & $-0.492 \pm 0.003$ & $0.713 \pm 0.003$ & $0.72 \pm 0.07$ \\
D & $0.354 \pm 0.003$ & $1.040 \pm 0.003$ & $0.40 \pm 0.06$ \\
G1 & $-0.142 \pm 0.02$ & $0.561 \pm 0.02$ & \\
G2 & -1.870 & 4.140 & \\
\hline
\end{tabular}

one of the other components. The influence of micro-lensing in the $A$ component seems to be the least.

\section{LENS MODEL AND LENS REDSHIFT}

The theoretical time delay between a pair of lensed images depends on the deflector mass profile through the adopted lens model parameters but also on the cosmological scalefactor. The latter is a function of the source redshift $z_{\mathrm{s}}$, the lens redshift $z_{1}$ and the cosmology (essentially the Hubble parameter $H_{0}$ and to a lesser extent on $\Omega_{\mathrm{m}}$ for the case of a flat Universe, see e.g. Schneider, Ehlers \& Falco 1992). Refsdal was the first to demonstrate that $H_{0}$ can be deduced from the expression of time delays between pairs of lensed images, as long as $z_{\mathrm{s}}, z_{1}$ and the lens model are known (Refsdal 1964a,b). For the case of H1413+117, the main lens is a very faint object $(F 160 \mathrm{~W}>20.52 \mathrm{mag}, F 180 M>22.18 \mathrm{mag}$, Chantry \& Magain 2007) for which the redshift lacks a final and reliable estimation so far. However, the Planck Collaboration has recently proposed a robust estimation for $H_{0}$ and $\Omega_{\mathrm{m}}$ based on full-mission Planck observations of temperature and polarization anisotropies of the cosmic microwave background radiation (Planck Collaboration XIII 2016). Therefore, adopting a lens model, the value $z_{\mathrm{s}}=2.558$ (e.g. Barbainis et al. 1997) for the redshift of the source, as well as a flat universe characterized by $\Omega_{\mathrm{m}}=0.308 \pm 0.012$ and $H_{0}=67.8 \pm$ $0.9 \mathrm{~km} \mathrm{~s}^{-1} \mathrm{Mpc}^{-1}$, the expression of the theoretical time delays only depends on $z_{1}$.

We propose then to update the value of $z_{1}$ by determining the optimal set of parameters which fits the available observable quantities, that is to say the four lensed image positions, the three flux ratios and the three time delays. Such an approach has already been carried out by Goicoechea \& Shalyapin (2010) for H1413+117 and they obtained $z_{1}=1.88_{-0.11}^{+0.09}$. We followed the same procedure but we used a more recent value for $H_{0}$ and the $\chi^{2} / \mathrm{MFLC} / \delta=30 /$ group I set of time delays derived in the present work (see Table 4). The lensed image positions come from HST wide field planetary camera (WFPC)/WFPC2 images (Turnshek et al. 1997) and are reported in Table 5. We have adopted the mid-IR flux ratios given in MacLeod et al. (2009) and reported in the third column of Table 5. Although the image fluxes are likely affected by micro-lensing (see e.g. Hutsemékers 1993; Chae et al. 2001; this work) and extinction (Turnshek et al. 1997), they claim that the $11 \mu \mathrm{m}$ observations which led to the mid-IR flux ratios should be very little affected by both these effects.

The lens scenario consists of a point-like source that is lensed by a combination of three components: 
Table 6. Modelling results and comparison with previous work. The parameters $b_{\mathrm{Gk}}, \Delta \alpha_{\mathrm{Gk}}$ and $\Delta \delta_{\mathrm{Gk}}$ are given in arcsec, and, $\theta_{e_{\mathrm{G} 1}}$ and $\theta_{\gamma_{\mathrm{ext}}}$ in degree.

\begin{tabular}{lccc}
\hline Parameter & $\begin{array}{c}\text { Goicoechea } \\
\text { et al. } \\
(2010)\end{array}$ & $\begin{array}{c}\text { This work } \\
\left(\chi^{2} / \text { MFLC } / \delta\right. \\
=30 / \text { group I })\end{array}$ & $\begin{array}{c}\text { This work } \\
\text { (idem }+ \\
\left.\text { relaxing } \sigma_{\Delta t_{\mathrm{AD}}}\right)\end{array}$ \\
\hline$\chi^{2}$ & $7.5 / 7$ & $3.20 / 3$ & $2.61 / 3$ \\
$\chi_{\text {image }}^{2}$ & - & 0.01 & 0.01 \\
$\chi_{\mathrm{flux}}^{2}$ & - & 0.94 & 0.71 \\
$\chi_{\text {delay }}^{2}$ & - & 0.82 & 0.46 \\
$\chi_{\mathrm{G} 1}^{2}$ & - & 1.42 & 1.43 \\
$b_{\mathrm{G} 1}$ & 0.68 & 0.56 & 0.56 \\
$\Delta \alpha_{\mathrm{G} 1}$ & -0.165 & -0.163 & -0.164 \\
$\Delta \delta_{\mathrm{G} 1}$ & 0.552 & 0.550 & 0.552 \\
$e_{\mathrm{G} 1}$ & 0.28 & 0.32 & 0.31 \\
$\theta_{e_{\mathrm{G} 1}}$ & -37.6 & -39.7 & -39.2 \\
$b_{\mathrm{G} 2}$ & 0.45 & 0.38 & 0.47 \\
$\Delta \alpha_{\mathrm{G} 2}$ & $\equiv-1.87$ & $\equiv-1.87$ & $\equiv-1.87$ \\
$\Delta \delta_{\mathrm{G} 2}$ & $\equiv 4.14$ & $\equiv 4.14$ & $\equiv 4.14$ \\
$\gamma_{\mathrm{ext}}$ & 0.11 & 0.13 & 0.12 \\
$\theta_{\gamma_{\mathrm{ext}}}$ & 45.4 & 44.7 & 46.1 \\
$z_{1}$ & 1.88 & 1.95 & 1.97 \\
\hline
\end{tabular}

(i) the main lensing galaxy G1 modelled as a singular isothermal ellipsoid (SIE) characterized by its relative position $\left(\Delta \alpha_{\mathrm{G} 1}, \Delta \delta_{\mathrm{G} 1}\right)$ with respect to the lensed image $A$, mass scale $b_{\mathrm{G} 1}$, ellipticity $e_{\mathrm{G} 1}$ and position angle $\theta_{e_{\mathrm{G} 1}}$,

(ii) the lensing galaxy G2 (object No. 14 in Kneib et al. 1998a) modelled as a singular isothermal sphere (SIS) characterized by its relative position $\left(\Delta \alpha_{\mathrm{G} 2}, \Delta \delta_{\mathrm{G} 2}\right)$ with respect to the lensed image $A$ and mass scale $b_{\mathrm{G} 2}$,

(iii) an external shear to account for the presence of galaxy overdensities and other contributions, characterized by its strength $\gamma_{\mathrm{ext}}$ and orientation $\theta_{\gamma_{\mathrm{ext}}}$.

Since the position of the gravity centre of the main lens is not precisely known, it has been considered as an additional parameter which is allowed to vary during the fit. The adopted G2 galaxy position with respect to the lensed image $A$ is $(\Delta \alpha, \Delta \delta)=$ $(-1.87,4.14)$ arcsec (Kneib et al. 1998a). Let us note that since we consider G1 and G2 to be lying in the same plane, the lens redshift $z_{1}$ refers to the pair G1-G2. Using the GRAVLENS ${ }^{1}$ software package (Keeton 2011), the best solutions for the $\chi^{2} / \mathrm{MFLC} / \delta$ $=30 /$ group I set of time delays (see Table 3) is $z_{1}=1.95_{-0.10}^{+0.06}$ with $\chi_{\text {tot }}^{2}=3.20$ for 3 degrees of freedom (dof). The model has 14 constraints (the four lensed image positions, the three flux ratios and the three time delays) and 11 parameters (see Table 6, plus the point-like source position). Contrary to MacLeod et al. (2009) and Goicoechea \& Shalyapin (2010), we do not assume any priors on the ellipticity and position of G1, and the strength of the external shear.

In Section 7, we found evidence of significant micro-lensing variability in the light curve of the component $D$. Such an effect could bias our determination of $\Delta t_{\mathrm{AD}}$. To quantify this impact on the determination of $z_{1}$, we relaxed the time delay constraint between $\mathrm{A}$ and $\mathrm{D}$, i.e. we did not take into account $\Delta t_{\mathrm{AD}}$, and obtained $z_{1}=1.97_{-0.11}^{+0.07}$ with $\chi_{\text {tot }}^{2}=2.61$ for dof $=3$.

In Fig. 13, we have illustrated the total $\chi_{\text {tot }}^{2}$ as a function of $z_{1}$ (thick solid line) both for the $\chi^{2} / \mathrm{MFLC} / \delta=30 /$ group I set of time delays with (right-hand panel) and without (left-hand panel) relaxing the time delay constraint on $D$. The total $\chi_{\text {tot }}^{2}$ has been broken down into four contributions, respectively for the lensed image positions $\left(\chi_{\text {image }}^{2}\right.$, dashed lines $)$, the flux ratios $\left(\chi_{\text {flux }}^{2}\right.$, dash-dotted lines $)$, the time delays ( $\chi_{\text {delay }}^{2}$, thin solid lines) and the G1 position $\left(\chi_{\mathrm{G} 1}^{2}\right.$, dotted lines), see the first part of Table 6 . The $1 \sigma$ (68 per cent) and $2 \sigma$ (95 per cent) confidence intervals are represented respectively by the dark and whole shaded areas. In both cases, the fit is dominated in the $1 \sigma$ confidence interval by the main galaxy $\mathrm{G} 1$ position. The contribution of $\chi_{\text {flux }}^{2}$ is also meaningful and clearly shapes the total $\chi_{\text {tot }}^{2}$ curve. Since the fit allows to reproduce almost perfectly the lensed image positions, the $\chi_{\text {image }}^{2}$ contribution to the total $\chi^{2}$ is almost negligible regardless of $z_{1}$. We have tested the goodness of the fit for lens redshift values down to 1 . Although the total $\chi_{\text {tot }}^{2}$ was not satisfactory, we noted that the lensed image positions were equally well reproduced. This tends to confirm that the lensed image positions can be easily reproduced with a large range of model parameters but also with different families of mass profiles. In particular, Schneider \& Sluse $(2013,2014)$ have theoretically pointed out the existence of a general transformation of the source plane, the so-called source-position transformation (SPT) whose well-known mass sheet degeneracy (MSD, Falco, Gorenstein \& Shapiro 1985) is a special case. The SPT defines a new family of deflection laws and leaves almost invariant the lensed image positions and their flux ratios. Since no complete quantitative analysis describing how the SPT affects the behaviour of the time delays exists yet, it is difficult to infer how it would impact on the value of the lens redshift. However, accounting for a uniform external convergence (MSD) characterized by $\kappa_{\text {ext }} \sim 0.1$, Goicoechea \& Shalyapin (2010) have estimated that the value of the lens redshift should increase by 3 per cent at most.

The lens redshift estimations obtained for the $\chi^{2} / \mathrm{MFLC} / \delta=$ $30 /$ group I set of time delays, with and without relaxing the time delay constraint on $\mathrm{D}$, are consistent. The improvement in $\chi_{\text {tot }}^{2}$ when relaxing the time delay constraint on $\mathrm{D}$ comes respectively from the time delays $\left(\Delta \chi_{\text {delay }}^{2}=0.36\right)$ and the flux ratios $\left(\Delta \chi_{\text {flux }}^{2}=0.23\right)$, see Table 6. Furthermore, the lens redshift estimations are also in good agreement with the value derived by Goicoechea \& Shalyapin (2010), $z_{1}=1.88_{-0.11}^{0.09}$, even though our results constitute a slight improvement in terms of reduced total $\chi_{\text {tot }}^{2}$. In addition, several arguments proposed in Goicoechea \& Shalyapin (2010) remain valid, that is to say that $z_{1}$ is in good or reasonable agreement with: (1) the G2 photometric redshift of about 2 derived by Kneib et al. (1998a); (2) the most distant detected overdensity at $z_{\text {ove }}=1.75 \pm$ 0.2 (Kneib et al. 1998a; Faure et al. 2004); (3) and absorbers at $z_{\text {abs }}$ $\sim 1.87$ (Turnshek et al. 1988). In addition, $z_{1} \sim 1.95$ is in agreement with the fact that the main galaxy G1 has been detected in $H S T /$ NICMOS-2 images with the near-IR $H$-band $F 160 W$ (bandwidth $=[1.4,1.8] \mu \mathrm{m})$ and $F 180 M($ bandwidth $=[1.76,1.83] \mu \mathrm{m})$ filters (Chantry \& Magain 2007) but not on HST/WFPC2 images, neither with the near-IR $I$-band $F 814 W$ (bandwidth $=[0.7,1] \mu \mathrm{m}$ ) nor the near-IR $R$-band $F 702 W$ (bandwidth $=[0.60 .8] \mu \mathrm{m}$ ) filters (Turnshek et al. 1997). Indeed, for $z_{1}=1.95$, the galaxy spectrum Ca II break ${ }^{2}$ occurs at $\lambda_{\text {break }} \sim 1.18 \mu \mathrm{m}$ while, for $z_{1}=1$, it occurs at $\lambda_{\text {break }} \sim 0.8 \mu \mathrm{m}$. Since the main galaxy G1 should be brighter at wavelengths $\lambda>\lambda_{\text {break }}$, it is clear that, for the case of $z_{1} \sim 1.95$, its detection would likely be easier with the $F 160 \mathrm{~W}$ and $F 180 M$ filters, both with $\lambda>1.18 \mu \mathrm{m}$, rather than with the $F 814 W$ and $F 702 W$ filters, both with $\lambda<1.18 \mu \mathrm{m}$. Conversely, a main galaxy G1 at $z_{1}=$ 

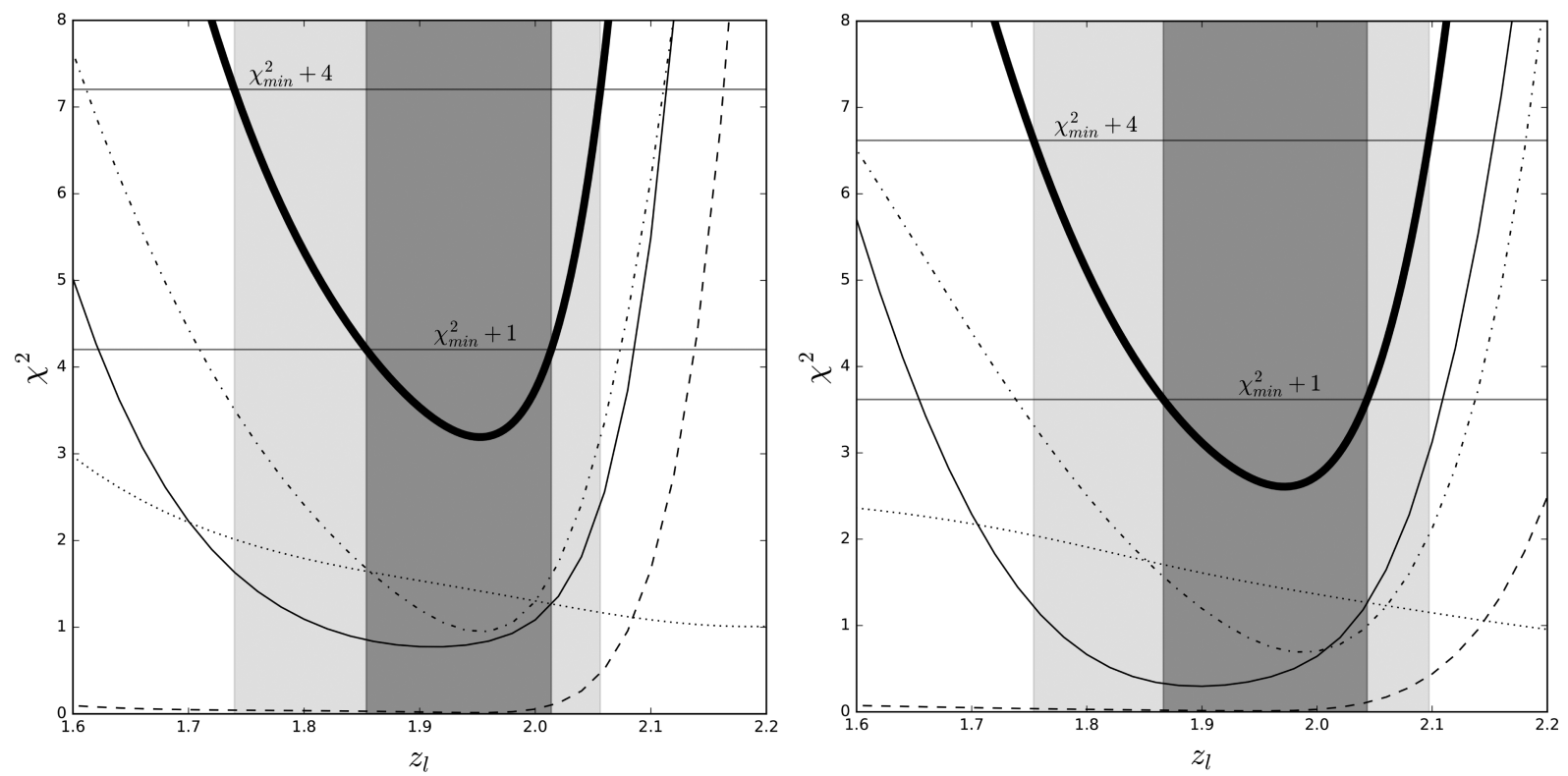

Figure 13. Estimation of the lens redshift $z_{1}$ for the $\chi^{2} / \mathrm{MFLC} / \delta=30 /$ group I set of time delays, without (left-hand panel) and with (right-hand panel) relaxing the time delay constraint on $D$. The total $\chi_{\text {tot }}^{2}$ (thick solid lines) has been broken down into four contributions, respectively $\chi_{\text {image }}^{2}$ (dashed lines), $\chi_{\text {flux }}^{2}$ (dash-dotted lines), $\chi_{\text {delay }}^{2}$ (thin solid lines) and $\chi_{\mathrm{G} 1}^{2}$ (dotted lines). We have also represented the $1 \sigma$ (dark shaded area) and $2 \sigma$ (whole shaded area) confidence intervals.

Table 7. Modelled image and G1 positions, flux ratios and time delays derived from the best solution respectively for the $\chi^{2} /$ MFLC $/ \delta=30$ /group I set of time delays, with or without relaxing the time delay constraint on $D$.

\begin{tabular}{|c|c|c|}
\hline Parameter & $\begin{array}{c}\text { This work } \\
\left(\chi^{2} / \mathrm{MFLC} / \delta=30 / \text { group I }\right)\end{array}$ & $\begin{array}{c}\text { This work } \\
\text { (idem }+ \text { relaxing } \sigma_{\Delta t_{\mathrm{AD}}} \text { ) }\end{array}$ \\
\hline \multicolumn{3}{|c|}{ Image positions (in arcsec) } \\
\hline A & {$[0.0,0.0]$} & {$[0.0,0.0]$} \\
\hline B & {$[0.744,0.168]$} & {$[0.744,0.168]$} \\
\hline $\mathrm{C}$ & {$[-0.492,0.713]$} & {$[-0.492,0.713]$} \\
\hline $\mathrm{D}$ & {$[0.354,1.040]$} & {$[0.354,1.040]$} \\
\hline \multicolumn{3}{|c|}{ Lens position (in arcsec) } \\
\hline G1 & {$[-0.163,0.550]$} & {$[-0.164,0.552]$} \\
\hline \multicolumn{3}{|l|}{ Flux ratios } \\
\hline A & $\equiv 1.0$ & $\equiv 1.0$ \\
\hline B & 0.81 & 0.79 \\
\hline $\mathrm{C}$ & 0.74 & 0.74 \\
\hline $\mathrm{D}$ & 0.45 & 0.42 \\
\hline \multicolumn{3}{|c|}{ Time delays (in days) } \\
\hline$\Delta t_{\mathrm{AB}}$ & -15.6 & -16.4 \\
\hline$\Delta t_{\mathrm{AC}}$ & -19.6 & -20.3 \\
\hline$\Delta t_{\mathrm{AD}}$ & 28.9 & 32.7 \\
\hline
\end{tabular}

1 would be already brighter at wavelengths $\lambda>0.8 \mu \mathrm{m}$, and would have likely been detected with the $F 814 W$ filter, which is actually not the case.

For both the $\chi^{2} / \mathrm{MFLC} / \delta=30$ /group I set of time delays with and without relaxing the time delay constraint on $\mathrm{D}$, the corresponding modelling results for the best solution are reported in the second part of Table 6 . The corresponding results for the modelled image positions, flux ratios and time delays are reported in Table 7. The relative position and position angle of G1 as well as the shear orientation are in very good agreement with the ones obtained by Goicoechea \& Shalyapin (2010). As mentioned in the latter paper, a weak lensing analysis performed by Faure et al. (2004) indicated a shear direction of $45^{\circ}$ and a shear strength smaller than 0.17 , which

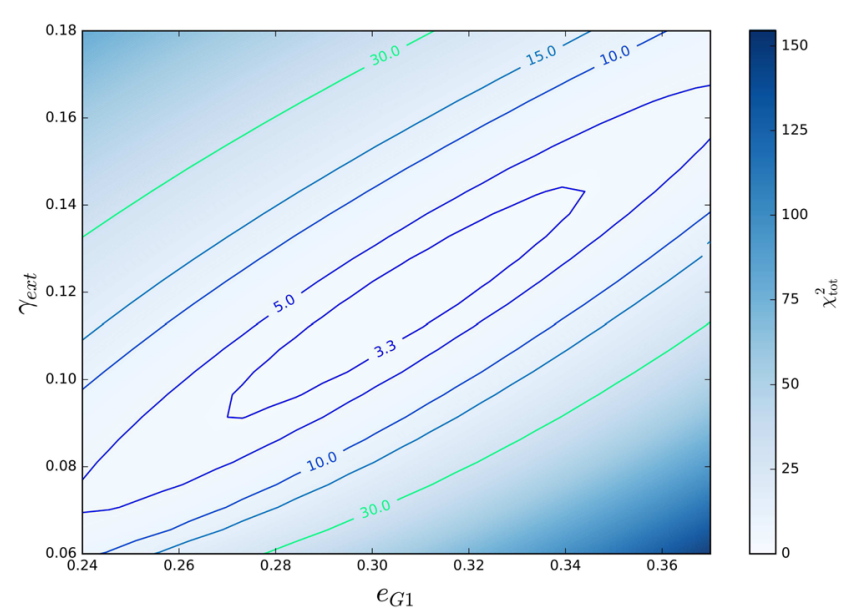

Figure 14. The total $\chi_{\text {tot }}^{2}$ map illustrating the degeneracy between two model parameters: the ellipticity of the main lens galaxy G1 and the strength of the external shear.

is in very good agreement with our modelling results, respectively $\left(\gamma_{\mathrm{ext}}, \theta_{\gamma_{\mathrm{ext}}}\right)=\left(0.13,44.7^{\circ}\right)$ for the $\chi^{2} / \mathrm{MFLC} / \delta=30 /$ group I set of time delays and $\left(\gamma_{\mathrm{ext}}, \theta_{\gamma_{\mathrm{ext}}}\right)=\left(0.12,46.1^{\circ}\right)$ when relaxing the time delay constraint on D. However, we note that while the G1 mass scale is significantly shorter, the external shear and the ellipticity are slightly larger than the ones given in Goicoechea \& Shalyapin (2010). Since the net quadrupole moment can have contributions from both the main lens galaxy ellipticity and the presence of an external shear, there might be a substantial degeneracy between these two parameters (Keeton et al. 1997). To point out this degeneracy, we have illustrated in Fig. 14 the total $\chi_{\text {tot }}^{2}$ map for ranges $e_{\mathrm{G} 1} \in$ $[0.24,0.37]$ and $\gamma_{\text {ext }} \in[0.06,0.18]$ which corresponds to the solution $z_{1}=1.97$. It is clear that our solution is fully compatible with the one given in Goicoechea \& Shalyapin (2010). Same results were obtained for our solution $z_{1}=1.95$. 


\section{SUMMARY AND CONCLUSIONS}

\subsection{Observations of $\mathbf{H 1 4 1 3 + 1 1 7}$}

In this article, we have presented for the first time an analysis of long-term photometric light curves derived for the lensed images of the quadruply imaged quasar H1413+117 (Clover Leaf). This object was observed in the $V$ and $R$ spectral bands with the $1.5-\mathrm{m}$ telescope of the Maidanak observatory from 2001 to 2008.

Due to the compactness of this GLS, it was very challenging to retrieve the photometric flux of its four individual lensed components. On the other hand, there is no need for taking into account the light contamination from the lensing galaxy, as its contribution to the total flux as a whole and for the individual components in the Maidanak CCD frames is negligibly small (of the order of $22 \mathrm{mag}$, Chantry \& Magain 2007).

\subsection{A very efficient photometric method}

To derive the magnitudes of the lensed QSO images, we have developed a new photometric technique: the adaptive PSF-fitting technique. First, this method only works with a numerical PSF and does not require an analytical representation. Secondly, there is no need for the assumption of the PSF invariance over large regions of the CCD frame. To calculate the fluxes of the lensed components, we assumed the PSF invariance over only the GLS (i.e. less than 2 arc$\mathrm{sec})$. The problem of deriving the fluxes of the lensed QSO images is reduced to solving a system of ordinary linear equations. The shape and width of the light distribution of the PSF do not really matter. Our method can also be successfully used for images performed in the defocused mode (Southworth et al. 2009, 2014).

\subsection{New light curves}

As a result of applying the adaptive PSF-fitting method to the Maidanak $\mathrm{H} 1413+117$ data set, we have provided new long-term photometric light curves of the lensed images. To determine the photometric error bars affecting each data point, we have simulated CCD images for each of these 500 frames including key factors such as: the Poisson noise due to the GLS, white noise for the sky background, uncertainties of the lensed image centring at the subpixel level, etc. The measurement of the error magnitudes of the lensed components corresponds to the standard deviation of the distribution of the results obtained for the various series of simulated frames.

\subsection{Brightness and colour variations}

We distinguished the light curves of the Clover Leaf lensed components in the $V$ and $R$ filters, and the $V-R$ colour curve. The light curves of the whole system and of the individual components correlate very well with each other. We see two types of variations: short-term (with amplitude $\sim 0.1 \mathrm{mag}$ ) during separate seasons and long-term (with an amplitude larger than $0.15 \mathrm{mag}$ ) over the timescale of several seasons. The colour changes are also characteristic of quasars [as in UM673, Koptelova et al. (2012)] - with an apparent flux decrease leading to some reddening, and vice versa - during an apparent flux increase, the system becomes bluer.

\subsection{Time delay calculations}

Our long-term photometric light curves have allowed us to estimate the time delays between the lensed images of the Clover Leaf. In the calculations, we separately relied on the seasons between 2003 and
2006 showing the most frequently sampled data. We did not use the whole light curves because of gaps between seasons with durations of several months. On the other hand, micro-lensing events may change the magnitude difference between the components (this in turn makes it impossible to use the light curves of the components in a general way).

This problem was solved with the help of different methods and approaches: we used chi-squared tests with linear interpolation and two different dispersion formulas. To estimate the errors of our time delay predictions, we generated synthetic light curves on the basis of previously calculated magnitudes from simulated CCD frames. As a second alternative method, we used the classical method of normal distribution of stellar magnitudes. Also, we considered separately the DLC and MFLC.

Fortunately, thanks to a significant increase in flux of all the components in 2004 (see Figs 9 and 10; JD = 3130-3150), we could obtain some relevant values for the time delays (Section 7). Our values for $\Delta t_{\mathrm{AB}}$ and $\Delta t_{\mathrm{AC}}$ are in full agreement with those obtained by Goicoechea \& Shalyapin (2010), whereas we derived a time delay between the $\mathrm{A}$ and $\mathrm{D}$ components $\left(\Delta t_{\mathrm{AD}}\right)$ that is not consistent with the previous one (using $1 \sigma$ confidence intervals). This discrepancy is likely due to an appreciable micro-lensing variation in the $R$-band light curve of the component D (see Sections 6 and 7).

\subsection{Micro-lensing events}

The complex and sometimes uncorrelated behaviours of the light and colour curves indicate some active intrinsic variability and continuous influence of micro-lensing. The micro-lensing rate measured for the A, B, C components, on average, corresponds to the characteristic rate of micro-lensing $10^{-4} \mathrm{mag} \mathrm{d}^{-1}$ reported for other known gravitationally lensed quasars (Gaynullina et al. 2005; Vakulik et al. 2006; Goicoechea \& Shalyapin 2010; Tsvetkova et al. 2010; Ricci et al. 2011).

However, we found an unusually strong influence of microlensing in the D component. In 2004, the light of this component has varied with a rate of $10^{-3} \mathrm{mag} \mathrm{d}^{-1}$ and almost reached the brightness of the $\mathrm{C}$ component in 2005 . We can assert that this is a recurrent process, as similar flux variations have been previously reported (Arnould et al. 1993; Remy et al. 1996).

Even more dramatic brightness changes of the lensed components due to micro-lensing have been reported for Q2237+0305 (Udalski et al. 2006; Koptelova et al. 2007). These findings support the view of Witt et al. (1995) about the general nature of quadrupole systems and the inevitability of micro-lensing events in them. Further evidence of the continuous influence of micro-lensing is the time variability of the magnitude difference between the lensed components, which vary over a very large range (Section 7).

\subsection{Lens model and lens redshift}

Following the lens scenario proposed in Goicoechea \& Shalyapin (2010), we have modelled the H1413+117 lens system with an SIE (for the lens galaxy G1), an SIS (for the lens galaxy G2) and an external shear (for galaxy overdensities and other contributions). Based on available observational constraints (resp. the lensed image positions, mid-IR flux ratios and two sets of time delays derived in the present work), we have improved the lens modelling and updated the estimation for the lens redshift: $z_{1}=1.95_{-0.10}^{+0.06}$ for the $\chi^{2} / \mathrm{MFLC} / \delta$ $=30 /$ group I set of time delays. We found a slight improvement in the $\chi_{\text {tot }}^{2}$ when we relax the time delay constraint on the component $\mathrm{D}$ but without significantly affecting the determination of the lens redshift value: $z_{1}=1.97_{-0.11}^{+0.07}$. These two estimations are in good 
agreement with the previous one derived in Goicoechea \& Shalyapin (2010). Due to the galaxy spectrum Ca II break feature, the main lens galaxy detection in $H S T /$ NICMOS-2 images (IR $H$-band $F 160 W$ and $F 180 M$ filters), but not in $H S T /$ WFPC2 images (IR $I$-band $F 814 W$ and IR $R$-band $F 702 W$ filters), seems to be more compatible with a high value for the lens redshift, $z_{1} \sim 1.95$, rather than $z_{1} \sim 1$.

As a recommendation, we think that the photometric adaptive PSF-fitting method which has been developed by us is very promising, since it can be successfully applied to a very wide class of CCD frames. This applies to images and CCD frames with different kinds of distortion.

We also provided accurate values of the time delays in $\mathrm{H} 1413+117$. On the other hand, due to the strong micro-lensing variability in the $\mathrm{D}$ component we should remain very careful about the use of the time delay values between the pairs of the $\mathrm{AD}, \mathrm{BD}$, $\mathrm{CD}$ components. It would be very nice to confirm these results from independent observations.

\section{ACKNOWLEDGEMENTS}

This research has been supported thanks to a Research Fellowship grant awarded by the Belgian Federal Science Policy Office (BELSPO). Observations carried out by UBAI researchers in 2003-2005 were supported by the German Research Foundation (DFG) grant 436 USB 113/5/0-1. JS acknowledges support from the Communauté française de Belgique - Actions de recherches concertées - Académie Wallonie - Europe. ASG thanks the Russian Science Foundation (project no. 14-22-00041) for support. The research of SAI MGU team is supported by Russian Foundation for Basic Research, grant 14-02-01274.

\section{REFERENCES}

Arnould P. et al., 1993, in Surdej J., Fraipont-Caro D., Gosset E., Refsdal S., Remy M., eds, Gravitational Lenses in the Universe. Université de Liège, Institut d'Astrophysique, Liège, p. 169

Artamonov B. P. et al., 2010, Astron. Rep., 54, 1019

Barbainis R., Maloney P., Antonucci R., Alloin D., 1997, ApJ, 484, 695

Chae K.-H., Turnshek D. A., 1999, ApJ, 514, 587

Chae K.-H., Turnshek D. A., Schulte-Ladbeck R. E., Rao S. M., Lupie O. L., 2001, ApJ, 561, 653

Chantry V., Magain P., 2007, A\&A, 470, 467

Chartas G., Eracleous M., Agol E., Gallagher S. C., 2004, ApJ, 606, 78

Drew J. E., Boksenberg A., 1984, MNRAS, 211, 813

Ehgamberdiev S. A., Baijumanov A. K., Ilyasov S. P., Sarazin M., Tillayev Y. A., Tokovinin A. A., Ziad A., 2000, A\&AS, 145, 293

Elyiv A., Melnyk O., Finet F., Pospieszalska-Surdej A., Chiappetti L., Pierre M., Sadibekova T., Surdej J., 2013, MNRAS, 434, 3305

Falco E. E., Gorenstein M. V., Shapiro I. I., 1985, ApJ, 289, L1

Faure C., Alloin D., Kneib J. P., Courbin F., 2004, A\&A, 428, 741

Gaynullina E. R. et al., 2005, A\&A, 440, 53

Gil-Merino R., Wisotzki L., Wambsganss J., 2002, A\&A, 381, 428

Goicoechea L. J., Shalyapin V. N., 2010, ApJ, 708, 995

Hazard C., Morton D. C., Terlevich R., McMahon R., 1984, ApJ, 282, 33

Hutsemékers D., 1993, A\&A, 280, 435

Hutsemékers D., Borguet B., Sluse D., Riaud P., Anguita T., 2010, A\&A, 519, A103

Jovanović P., 2005, Memor. Soc. Astron. Ital. Sup., 7, 56

Kayser R., Surdej J., Condon J. J., Kellerman K. I., Magain P., Remy M., Smette A., 1990, ApJ, 364, 15

Keel W. C., 2007, The Road to Galaxy Formation. Praxis Publishing Ltd, Chichester

Keeton C. R., 2011, Astrophysics Source Code Library, record ascl:1102.003 Keeton C. R., Kochanek C. S., Seljak U., 1997, ApJ, 482, 604

Kneib J.-P., Alloin D., Mellier Y., Guilloteau S., Barvainis R., Antonucci R., 1998a, A\&A, 329, 827
Kneib J.-P., Alloin D., Pello R., 1998b, A\&A, 339, L65

Koptelova E., Shimanovskaya E., Artamonov B., Sazhin M., Yagola A., Bruevich V., Burkhonov O., 2005, MNRAS, 356, 323

Koptelova E., Shimanovskaya E., Artamonov B., Yagola A., 2007, MNRAS, 381,1655

Koptelova E. et al., 2012, A\&A, 544, id. A51

Kundic T. et al., 1997, ApJ, 482, 75

Lupton R., Gunn J. E., Ivezić Z., Knapp G. R., Kent S., 2001, in Harnden F. R., Jr, Primini F. A., Payne H. E., eds, ASP Conf. Ser. Vol. 238, Astronomical Data Analysis Software and Systems X. Astron. Soc. Pac., San Francisco, p. 269

MacLeod C. L., Kochanek C. S., Agol E., 2009, ApJ, 699, 1578

Magain P., Surdej J., Swings J.-P., Borgeest U., Kayser R., 1988, Nature, 334,325

Magain P., Courbin F., Sohy S., 1998, ApJ, 494, 472

Massey P., Davis L. E., 1992, A User's Guide to Stellar CCD Photometry with IRAF. Available at: http://iraf.noao.edu/docs/docmain.html

Monier E. M., Turnshek D. A., Lupie O. L., 1988, ApJ, 496, 177

Pelt J., Kayser R., Refsdal S., Schramm T., 1996, A\&A, 305, 97

Planck Collaboration XIII, 2016, A\&A, 594, A13

Press W. H., Teukolsky S. A., Vetterling W. T., Flannery B. P., 1992, Numerical Recipes in C. The Art of Scientific Computing, 2nd edn. Cambridge Univ. Press, Cambridge

Refsdal S., 1964a, MNRAS, 128, 295

Refsdal S., 1964b, MNRAS, 128, 307

Remy M., Gosset E., Hutsemékers D., Revenaz B., Surdej J., 1996, in Kochanek C. S., Hewitt J. N., eds, Proc. IAU Symp. 173, Astrophysical Applications of Gravitational Lensing. Kluwer, Dordrecht, p. 261

Ricci D. et al., 2011, A\&A, 528, A42

Ricci D. et al., 2013, A\&A, 551, A104

Schneider P., Sluse D., 2013, A\&A, 559, A37

Schneider P., Sluse D., 2014, A\&A, 564, A103

Schneider P., Ehlers J., Falco E., 1992, Gravitational Lenses. SpringerVerlag, Berlin

Southworth J. et al., 2009, MNRAS, 396, 1023

Southworth J. et al., 2014, MNRAS, 444, 776

Stetson P. B., Davis L. E., Crabtree D. R., 1990, in Jacoby G.H., eds., Proc. ASP Conf., 8, Tucson, AZ, p. 289

Tsvetkova V. S. et al., 2010, MNRAS, 406, 2764

Turnshek D. A., Foltz C. B., Grillmair C. J., Weymann R. J., 1988, ApJ, 325,651

Turnshek D. A., Lupie O. L., Rao S. M., Espey B. R., Sirola C. J., 1997, ApJ, 485, 100

Udalski A. et al., 2006, Acta Astron., 56, 307

Ullán A., Goicoechea L. J., Zheleznyak A. P., Koptelova E., Bruevich V. V., Akhunov T., Burkhonov O., 2006, A\&A, 452, 25

Vakulik V., Schild R., Dudinov V., Nuritdinov S., Tsvetkova V., Burkhonov O., Akhunov T., 2006, A\&A, 447, 905

Wambsganss J., Paczynski B., 1991, AJ, 102, 864

Witt H. J., Mao S., Schechter P. L., 1995, ApJ, 443, 18

\section{SUPPORTING INFORMATION}

Supplementary data are available at MNRAS online.

Appendix A. Photometry of $\mathrm{H} 1413+117$ in the $R$ and $V$ bands. Only a portion of the table is shown here for guidance regarding its form and content.

Appendix B. Calculation of the photometric error bars for the four lensed components of $\mathrm{H} 1413+117$.

Please note: Oxford University Press is not responsible for the content or functionality of any supporting materials supplied by the authors. Any queries (other than missing material) should be directed to the corresponding author for the article.

This paper has been typeset from a $\mathrm{T}_{\mathrm{E}} \mathrm{X} / \mathrm{LAT} \mathrm{E} \mathrm{X}$ file prepared by the author. 